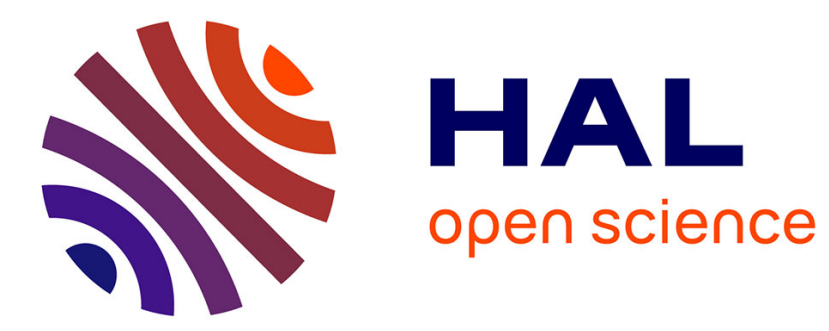

\title{
Convexity of graph-restricted games induced by minimum partitions
}

Alexandre Skoda

\section{To cite this version:}

Alexandre Skoda. Convexity of graph-restricted games induced by minimum partitions. RAIRO Operations Research, 2019, 53 (3), pp.841-866. 10.1051/ro/2017069 . halshs-01617023

\section{HAL Id: halshs-01617023 \\ https://shs.hal.science/halshs-01617023}

Submitted on 16 Oct 2017

HAL is a multi-disciplinary open access archive for the deposit and dissemination of scientific research documents, whether they are published or not. The documents may come from teaching and research institutions in France or abroad, or from public or private research centers.
L'archive ouverte pluridisciplinaire HAL, est destinée au dépôt et à la diffusion de documents scientifiques de niveau recherche, publiés ou non, émanant des établissements d'enseignement et de recherche français ou étrangers, des laboratoires publics ou privés. 


\title{
Convexity of Graph-Restricted Games Induced by Minimum Partitions
}

\author{
A. Skoda*
}

August 10, 2017

\begin{abstract}
We consider restricted games on weighted graphs associated with minimum partitions. We replace in the classical definition of Myerson restricted game the connected components of any subgraph by the subcomponents corresponding to a minimum partition. This minimum partition $\mathcal{P}_{\min }$ is induced by the deletion of the minimum weight edges. We provide five necessary conditions on the graph edge-weights to have inheritance of convexity from the underlying game to the restricted game associated with $\mathcal{P}_{\text {min }}$. Then, we establish that these conditions are also sufficient for a weaker condition, called $\mathcal{F}$-convexity, obtained by restriction of convexity to connected subsets. Moreover, we prove that inheritance of convexity for Myerson restricted game associated with a given graph $G$ is equivalent to inheritance of $\mathcal{F}$-convexity for the $\mathcal{P}_{\min }$-restricted game associated with a particular weighted graph $G^{\prime}$ built from $G$ by adding a dominating vertex, and with only two different edge-weights. Then, we prove that $G$ is cycle-complete if and only if a specific condition on adjacent cycles is satisfied on $G^{\prime}$.
\end{abstract}

Keywords: communication networks, cooperative game, convex game, restricted game, partitions.

\section{Introduction}

A cooperative game is a pair $(N, v)$ where $N$ is a set of players, and $v$ is the characteristic function of the game which assigns to any coalition $A \subseteq N$ a value $v(A) \in \mathbb{R}$, and satisfies $v(\emptyset)=0$. We consider an arbitrary correspondence $\mathcal{P}$ which associates to every subset $A \subseteq N$ a partition $\mathcal{P}(A)$ of $A$. Then, for every game $(N, v)$, we define the $\mathcal{P}$-restricted game $(N, \bar{v})$ by:

$$
\bar{v}(A)=\sum_{F \in \mathcal{P}(A)} v(F), \text { for all } A \subseteq N .
$$

*Corresponding author. Université de Paris I, Centre d'Economie de la Sorbonne, 106-112 Bd de l'Hôpital, 75013 Paris, France. E-mail: alexandre.skoda@univ-paris1.fr 
Through many concrete choices for the correspondence $\mathcal{P}$, the new game $(N, \bar{v})$ can take into account many combinatorial structures and different aspects of cooperation restrictions.

A founding example is the Myerson's correspondence $\mathcal{P}_{M}$ associated with communication games (Myerson, 1977). A communication game is a combination of a cooperative game $(N, v)$ and an undirected graph $G=(N, E)$ representing communication possibilities between the players. For every coalition $A \subseteq N$, we denote by $G_{A}$ the graph $(A, E(A))$ where $E(A)=\{e \in$ $E \mid e=\{i, j\}$ with $i, j \in A\}$. $\mathcal{P}_{M}(A)$ is the set of connected components of $G_{A}$. Then, the $\mathcal{P}_{M}$-restricted game $(N, \bar{v})$, known as Myerson restricted game, takes into account the connectivity between players in $G$. It is considered that only connected coalitions are able to cooperate and get their initial values. Many other correspondences have been considered to define restricted games (see, e.g., Algaba et al. (2001); Bilbao (2000, 2003); Faigle (1989); Grabisch and Skoda (2012); Grabisch (2013)).

We say that a correspondence $\mathcal{P}$ satisfies inheritance of superadditivity (resp. convexity) if for all superadditive (resp. convex) games $(N, v)$, the $\mathcal{P}$-restricted games $(N, \bar{v})$ are superadditive (resp. convex). A characterization of correspondences satisfying inheritance of superadditivity has been established in (Grabisch and Skoda, 2012). Another one for inheritance of convexity, but with the family of underlying games $(N, v)$ restricted to the family of unanimity games, has also been proved in (Grabisch and Skoda, 2012). In the case of communication games, these characterizations imply strong conditions on the communication graphs. In particular, we prove in this paper that they imply the graph characterization established in (van den Nouweland and Borm, 1991) for the correspondence $\mathcal{P}_{M}$ : there is inheritance of convexity for $\mathcal{P}_{M}$ if and only if the communication graph is cycle-complete $^{1}$. Of course, an abstract theory of restricted games associated with correspondences must be developed in relation with many other concrete examples of correspondences.

We consider in this paper cooperative games with cooperation restrictions described by a weighted graph. A weighted graph is a graph in which each edge $e \in E$ has been assigned a real number $w(e)$, called the weight of $e$. Weighted graphs can be used to represent various types of networks, e.g., communication, transportation, or social networks. The interpretation of edge-weights depends on the context. For a communication network, edge-weights can correspond to capacities or communication levels between players. In the case of a social network, edge-weights can be values reflecting intensities of players' relationships. Then it is likely that, in a given coalition, players are more or less prone to cooperate depending on the weights of their links. Moreover, it may be the case that the existence of links between

\footnotetext{
${ }^{1} \mathrm{~A}$ graph $G=(N, E)$ is cycle-complete if for any cycle $C=\left(v_{1}, e_{1}, v_{2}, e_{2}, \ldots, e_{m}, v_{1}\right)$ in $G$, the subset $\left\{v_{1}, v_{2}, \ldots, v_{m}\right\} \subseteq N$ of vertices of $C$ induces a complete subgraph in $G$.
} 
players is not sufficient to activate their cooperation. We will consider that two players have a privileged relation in a given coalition $A$ if they are linked by a non-minimum weight edge in $G_{A}$. Moreover, we will assume that only players with privileged relations cooperate. Therefore, we consider in this paper the correspondence $\mathcal{P}_{\text {min }}$ which associates to any coalition $A \subseteq N$ the partition of $A$ obtained by the removal of all minimum weight edges in $G_{A}$. Hence, we obtain a partition restricted game $(N, \bar{v})$ where connectedness is not sufficient to have the cooperation of players.

In this framework, we study necessary conditions on weighted graphs under which $\mathcal{P}_{\min }$ satisfies inheritance of convexity. We obtain five necessary conditions on the edge-weights of paths, stars, cycles, pans ${ }^{2}$ and adjacent cycles of the graph $G$. These conditions look like convexity conditions on the edge-weights. For instance, if the edge-weights of a path are all greater or equal to the weight of its first edge, then they have to be nondecreasing alongside the path. To establish these five necessary conditions, we only need to consider connected subsets. Hence, these necessary conditions are valid if we only assume inheritance of $\mathcal{F}$-convexity which is a weaker condition than convexity introduced in (Grabisch and Skoda, 2012) and obtained by restricting the definition of convexity to connected subsets. Then, we prove that these five conditions characterize inheritance of $\mathcal{F}$-convexity for the correspondence $\mathcal{P}_{\min }$ and this constitutes the main result of the paper (Theorem 15). Moreover, we fully describe the graphs satisfying these five necessary conditions.

The particular case of cycle-free graphs has already been investigated in (Grabisch and Skoda, 2012). Two weaker versions of the necessary conditions on paths and stars were established, and it was proved that they ensure inheritance of $\mathcal{F}$-convexity in this case. It was also observed that, for arbitrary graphs, a condition on edge-weights of cycles was required. In the present paper, these three conditions are extended to a more general setting: paths, stars, and cycles do not necessarily correspond to induced subgraphs.

Of course $\mathcal{F}$-convexity does not imply convexity but we think this notion is of interest in the context of communication games. Convexity corresponds to the idea that tendency for a player to join a coalition increases as the coalition grows. But if the coalition does not correspond to a connected subset, then this property seems rather unlikely. Indeed, the player will not be able to communicate with the whole group of players in the coalition, and in the worst case could be not even linked to any player in the coalition. If we consider that players should be at least able to communicate to cooperate, then it becomes natural to restrict convexity to connected subsets. Many other extensions of the notion of convexity have been considered in the literature (see, e.g., Edmonds and Giles (1977), Faigle (1989), Fujishige (2005)), where

\footnotetext{
${ }^{2} \mathrm{~A}$ pan graph is a graph obtained by joining a cycle to a vertex by an edge.
} 
the convexity has to be satisfied only for specific restricted families of subsets. Moreover, in a forthcoming paper (Skoda, 2017), we have completely classified the weighted graphs satisfying inheritance of convexity for $\mathcal{P}_{\min }$. Those graphs have strong restrictions on their combinatorial structures and on their edge-weights. In particular, they can have only three different edgeweights. In contrast, inheritance of $\mathcal{F}$-convexity allows an arbitrary number of edge-weights. We present at the end of the paper some results established in (Skoda, 2017) as they allow to highlight other major differences between graphs satisfying inheritance of convexity and the ones satisfying inheritance of $\mathcal{F}$-convexity.

An interesting feature of the $\mathcal{P}_{\text {min }}$-restricted game is that it can easily be linked to Myerson restricted game. More precisely, we establish that the Myerson restricted game associated with an arbitrary graph $G$ corresponds to a restriction of the $\mathcal{P}_{\min }$-restricted game associated with a particular weighted graph $G^{\prime}$ built from $G$ by simply adding a dominating vertex ${ }^{3}$, and with only two different edge-weights. This also provides an interesting link between convexity and $\mathcal{F}$-convexity. We prove that inheritance of convexity for the Myerson correspondence $\mathcal{P}_{M}$ on $G$ is equivalent to inheritance of $\mathcal{F}$ convexity for the correspondence $\mathcal{P}_{\min }$ on $G^{\prime}$. Moreover, we show that, due to the specific structure of $G^{\prime}$, and as there are only two edge-weights on $G^{\prime}$, the correspondence $\mathcal{P}_{\min }$ satisfies inheritance of $\mathcal{F}$-convexity on $G^{\prime}$ if and only if $G^{\prime}$ satisfies the condition on adjacent cycles. Hence, this last condition is particularly relevant as it is still required, whereas the other four necessary conditions are always satisfied on this particular class of weighted graphs. Furthermore, the previous result establishes a link between the condition on adjacent cycles and the characterization in (van den Nouweland and Borm, 1991), as it implies that the condition on adjacent cycles of the graph $G^{\prime}$ is equivalent to the cycle-completeness of the graph $G$.

Let us note that our characterization can lead to a practical way to check inheritance of $\mathcal{F}$-convexity for the correspondence $\mathcal{P}_{\text {min }}$. Of course, directly checking the five necessary and sufficient conditions would not result in a polynomial time algorithm as we would need to consider an exponential number of paths, stars, cycles, and pans. But we proved in a forthcoming paper (Skoda, 2016) that we only have to consider a polynomial number of specific paths and cycles associated with a minimum weight spanning tree. Then, we are able to construct a polynomial time algorithm to decide the inheritance of $\mathcal{F}$-convexity for $\mathcal{P}_{\text {min }}$, though the problem looks a priori highly non-polynomial. The complete proof of this result is too long to be included in this paper which is focused on providing a characterization of inheritance of $\mathcal{F}$-convexity for $\mathcal{P}_{\text {min }}$.

The article is organized as follows. In Section 2, we give preliminary definitions and recall results established in (Grabisch and Skoda, 2012). In

\footnotetext{
${ }^{3}$ A dominating vertex of a graph $G$ is a vertex adjacent to all other vertices of $G$.
} 
particular, we recall the definition of a correspondence, the definition of $\mathcal{F}$-convexity, and general conditions on a correspondence to have inheritance of convexity and $\mathcal{F}$-convexity. The $\mathcal{P}_{\text {min }}$-restricted game is defined in Section 3 . We provide a very simple counter-example to inheritance of convexity for $\mathcal{P}_{\text {min }}$ with a graph with only two different edge-weights, for which, nevertheless, inheritance of $\mathcal{F}$-convexity is satisfied. Section 4 describes the relations between the Myerson restricted game associated with a given graph $G$ and the restriction of the $\mathcal{P}_{\min }$-restricted game associated with a particular weighted graph $G^{\prime}$ built from $G$. Section 5 includes the main results ot the paper. In Section 5.1, we precisely describe the five conditions on edge-weights, and we establish that they are necessary to have inheritance of $\mathcal{F}$-convexity for the correspondence $\mathcal{P}_{\text {min }}$. Then, we prove that these conditions are also sufficient for superadditive games in Section 5.2. As a consequence, one only needs to verify inheritance of $\mathcal{F}$-convexity for unanimity games. That is an interesting and non-trivial result as a convex or $\mathcal{F}$-convex game is not in general a convex combination of unanimity games. In Section 5.3, we prove that cycle-completeness of a graph is satisfied if and only if one of the necessary conditions on adjacent cycles is satisfied on the particular graph described in Section 4. As a consequence, we reobtain the characterization of van den Nouweland and Borm (1991) for inheritance of convexity for the Myerson correspondence $\mathcal{P}_{M}$. In Section 5.4, we give a description of the class of graphs satisfying the necessary and sufficient conditions for inheritance of $\mathcal{F}$-convexity. This class is large as the number of different edge-weights and of adjacent cycles is not restricted. We finally state in Section 6 characterizations of inheritance of convexity. We conclude with some remarks and suggestions for generalization of these results to other correspondences in Section 7.

\section{Preliminary definitions and results}

Let $N$ be a given set. We denote by $2^{N}$ the set of all subsets of $N$. A game $(N, v)$ is zero-normalized if $v(i)=0$ for all $i \in N$. We recall that a game $(N, v)$ is superadditive if, for all $A, B \in 2^{N}$ such that $A \cap B=\emptyset$, $v(A \cup B) \geq v(A)+v(B)$. For any given subset $\emptyset \neq S \subseteq N$, the unanimity game $\left(N, u_{S}\right)$ is defined by:

$$
u_{S}(A)= \begin{cases}1 & \text { if } A \supseteq S, \\ 0 & \text { otherwise. }\end{cases}
$$

We note that $u_{S}$ is superadditive for all $S \neq \emptyset$. Let $X$ and $Y$ be two given sets. A correspondence $f$ with domain $X$ and range $Y$ is a map that associates to every element $x \in X$ a subset $f(x)$ of $Y$, i.e., a map from $X$ to $2^{Y}$. In this work we consider a specific correspondence $\mathcal{P}$ with domain and range $2^{N}$, such that for every subset $\emptyset \neq A \subseteq N$, the family $\mathcal{P}(A)$ of subsets 
of $N$ corresponds to a partition of $A$ (we set $\mathcal{P}(\emptyset)=\{\emptyset\}$ ). Let $(N, v)$ be a given game. Then the partition restricted game $(N, \bar{v})$ associated with $\mathcal{P}$ is defined by:

$$
\bar{v}(A)=\sum_{F \in \mathcal{P}(A)} v(F), \text { for all } A \subseteq N .
$$

We will more simply refer to this game as the $\mathcal{P}$-restricted game. Let us note that if $(N, v)$ is a communication game associated with a graph $G=(N, E)$ and if $\mathcal{P}$ corresponds to the partition into connected components, then $(N, \bar{v})$ is Myerson restricted game $\left(N, v^{M}\right)$ (Myerson, 1977).

For two given subsets $A$ and $B$ of $N, \mathcal{P}(A)$ is a refinement of $\mathcal{P}(B)$ if every block of $\mathcal{P}(A)$ is a subset of some block of $\mathcal{P}(B)$. The following result established in (Grabisch and Skoda, 2012) gives general conditions on a correspondence $\mathcal{P}$ to have inheritance of superadditivity.

Theorem 1. Let $N$ be an arbitrary set and $\mathcal{P}$ a correspondence on $N$. Then the following claims are equivalent:

1) For all $\emptyset \neq S \subseteq N$, the $\mathcal{P}$-restricted game $\left(N, \overline{u_{S}}\right)$ is superadditive.

2) For all subsets $A \subseteq B \subseteq N, \mathcal{P}(A)$ is a refinement of the restriction of $\mathcal{P}(B)$ to $A$.

3) For all superadditive game $(N, v)$ the $\mathcal{P}$-restricted game $(N, \bar{v})$ is superadditive.

Let $\mathcal{F}$ be a weakly union-closed family 4 of subsets of $N$ such that $\emptyset \notin \mathcal{F}$. A game $v$ on $2^{N}$ is said to be $\mathcal{F}$-convex if for all $A, B \in \mathcal{F}$ such that $A \cap B \in \mathcal{F}$, we have:

$$
v(A \cup B)+v(A \cap B) \geq v(A)+v(B) .
$$

Note that as $\mathcal{F}$ is weakly-union closed and $\emptyset \notin \mathcal{F}, A \cap B \in \mathcal{F}$ implies $A \cup B \in \mathcal{F}$. Of course convexity implies $\mathcal{F}$-convexity which implies also the following condition. If a game $v$ on $2^{N}$ is $\mathcal{F}$-convex, then for all $i \in N$ and all $A \subseteq B \subseteq N \backslash\{i\}$ such that $A, B$ and $A \cup\{i\} \in \mathcal{F}$, we have:

$$
v(B \cup\{i\})-v(B) \geq v(A \cup\{i\})-v(A) .
$$

Of course, if $\mathcal{F}=2^{N} \backslash \emptyset$ and if the game is superadditive then $\mathcal{F}$-convexity implies convexity and it is well known that there is equivalence of the two previous conditions (Schrijver, 2003). Let us note that $\left(N, u_{S}\right)$ is convex for all $S \neq \emptyset$. Let $(N, v)$ be a cooperative game associated with a graph

\footnotetext{
${ }^{4} \mathcal{F}$ is weakly union-closed if $A \cup B \in \mathcal{F}$ for all $A, B \in \mathcal{F}$ such that $A \cap B \neq \emptyset$ Faigle et al. (2010). Weakly union-closed families were introduced and analysed in Algaba (1998); Algaba et al. (2000) and called union stable systems.
} 
$G=(N, E)$. We say that a subset $\emptyset \neq A \subseteq N$ is connected if the induced graph $G_{A}=(A, E(A))$ is connected. The family of connected subsets of $N$ is obviously weakly union-closed (Algaba et al., 2000). For this last family the two previous conditions are also equivalent (Grabisch and Skoda, 2012).

Theorem 2. Let $G=(N, E)$ be an arbitrary graph and let $\mathcal{F}$ be the family of connected subsets of $N$. Then the following conditions are equivalent:

$v$ is $\mathcal{F}$-convex.

$$
\begin{aligned}
& v(B \cup\{i\})-v(B) \geq v(A \cup\{i\})-v(A), \text { for all } i \in N, \\
& \text { for all } A \subseteq B \subseteq N \backslash\{i\} \text { with } A, B, \text { and } A \cup\{i\} \in \mathcal{F} \text {. }
\end{aligned}
$$

The next theorem established in (Grabisch and Skoda, 2012) gives general abstract conditions on a correspondence $\mathcal{P}$ to have inheritance of convexity for the family of unanimity games.

Theorem 3. Let $N$ be an arbitrary set, $\mathcal{P}$ a correspondence on $N$, and $\mathcal{F}$ a weakly-union closed family of subsets of $N$ such that $\emptyset \notin \mathcal{F}$. If for all nonempty subset $S \subseteq N,\left(N, \overline{u_{S}}\right)$ is superadditive, then the following conditions are equivalent.

1) For all non-empty subset $S \subseteq N$, the game $\left(N, \overline{u_{S}}\right)$ is $\mathcal{F}$-convex.

2) For all $A, B \in \mathcal{F}$ such that $A \cap B \in \mathcal{F}, \mathcal{P}(A \cap B)=\left\{A_{j} \cap B_{k} \mid A_{j} \in\right.$ $\left.\mathcal{P}(A), B_{k} \in \mathcal{P}(B), A_{j} \cap B_{k} \neq \emptyset\right\}$.

Moreover if $\mathcal{F}=2^{N} \backslash\{\emptyset\}$ or if $\mathcal{F}$ corresponds to the set of all connected subsets of a graph then 1) and 2) are equivalent to:

3) For all $i \in N$ and for all $A \subseteq B \subseteq N \backslash\{i\}$ with $A, B$, and $A \cup\{i\} \in \mathcal{F}$, we have for all $A^{\prime} \in \mathcal{P}(A \cup\{i\}), \mathcal{P}(A)_{\mid A^{\prime}}=\mathcal{P}(B)_{\mid A^{\prime}}$.

Remark 1. We will mostly use Condition 3 of Theorem 3 in subsequent proofs with the family $\mathcal{F}$ of connected subsets.

\section{$3 \quad \mathcal{P}_{\min }$-restricted game}

Let $G=(N, E, w)$ be an arbitrary graph with an edge-weight function $w$ which assigns a weight $w(e)$ to each edge $e \in E$. We assume that the edge-weights are not all equal to avoid trivial situations. For any subset $A \subseteq N$, we denote by $\sigma(A)$ the minimum edge-weight in $G_{A}$, i.e., $\sigma(A)=$ $\min _{e \in E(A)} w(e)$, and by $\Sigma(A)$ the subset of edges of minimum weight in $E(A)$. Let $\mathcal{P}_{\text {min }}$ be the correspondence which associates to every subset $A \subseteq N$ the partition $\mathcal{P}_{\min }(A)$, the elements of which are the components of 
the graph $(A, E(A) \backslash \Sigma(A))$. We set $\mathcal{P}_{\min }(\emptyset)=\{\emptyset\}$. Then, for every game $(N, v)$, the $\mathcal{P}_{\text {min }}$-restricted game $(N, \bar{v})$ is defined by:

$$
\bar{v}(A)=\sum_{F \in \mathcal{P}_{\min }(A)} v(F), \text { for all } A \subseteq N .
$$

The $\mathcal{P}_{\min }$-restricted game takes into account the connectivity between players, but connectivity is not a sufficient condition to have cooperation of all players in a given coalition. As we delete the minimum weight edges there must also be some privileged relation between players to activate their cooperation. Hence, the $\mathcal{P}_{\text {min }}$-restricted game is very different from Myerson restricted game as shown in the following example. Let us consider the weighted graph $G$ represented in Figure 1 and the subsets $A=\{1,2,3\}$ and $B=\{1,2,4\}$. As $A$ and $B$ are connected subsets in $G$, they get with Myerson restricted game their initial values, i.e., $v^{M}(A)=v(A)$ and $v^{M}(B)=v(B)$. Although in both cases the three players can communicate as they form a complete subgraph of $G$, the result is different with the $\mathcal{P}_{\min }$-restricted game. Indeed, in the first case the edge-weights imply that only players 2 and 3 have a privileged relation and will cooperate. Therefore, they form a subcoalition and $\bar{v}(A)=v(\{1\})+v(\{2,3\})$. In the second case the three players have the same level of relationship. Therefore, they will not cooperate and $\bar{v}(B)=v(\{1\})+v(\{2\})+v(\{4\})$.

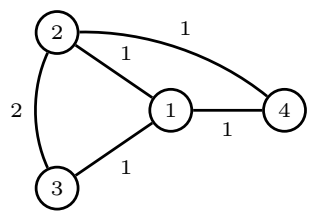

Figure 1: A weighted graph.

Moreover, let us note that, if the underlying game $(N, v)$ is superadditive, then the value obtained by a coalition with the $\mathcal{P}_{\min }$-restricted game $(N, \bar{v})$ is always less or equal to the value obtained with Myerson restricted game. Hence, the $\mathcal{P}_{\min }$-restricted game can be considered as a pessimistic alternative to Myerson restricted game. Although different from the $\mathcal{P}_{\text {min }^{-}}$ restricted game, we show in Section 4 that Myerson restricted game is closely linked to a particular case of the $\mathcal{P}_{\min }$-restricted game.

We can immediately observe that the correspondence $\mathcal{P}_{\min }$ satisfies inheritance of superadditivity. Indeed, as for all $A \subseteq B \subseteq N, \mathcal{P}_{\min }(A)$ is a refinement of $\mathcal{P}_{\min }(B)_{\mid A}$, Theorem 1 implies the following result.

Proposition 4. Let $G=(N, E, w)$ be an arbitrary weighted graph. Then, for every superadditive game $(N, v)$, the $\mathcal{P}_{\min }$-restricted game $(N, \bar{v})$ is superadditive. 
In comparison inheritance of convexity is very intricate. As pointed out in the introduction, we will restrict the study in this paper to inheritance of $\mathcal{F}$-convexity. We end this section with a counterexample to inheritance of convexity. Let us consider the graph represented in Figure 2. We suppose:

$$
w_{1}=w_{2}<w_{3}=w_{4} \text {. }
$$

We consider $i=1, A_{1}=\{2\}, A_{2}=\{4,5\}, A=\{2,4,5\}$, and $B=\{2,3,4,5\}$. Then $\bar{v}(B \cup\{i\})-\bar{v}(B)=v(i)$ and $\bar{v}(A \cup\{i\})-\bar{v}(A)=v\left(A_{2}\right)+v(i)-v(4)-$ $v(5)$. Taking $v=u_{A_{2}}$ we get $\bar{v}(B \cup\{i\})-\bar{v}(B)=0<1=\bar{v}(A \cup\{i\})-\bar{v}(A)$. Therefore there is no inheritance of convexity. It can easily be checked that if $A$ is replaced by any connected subset of $B$ then $\bar{v}(B \cup\{i\})-\bar{v}(B)=$ $v(i)=\bar{v}(A \cup\{i\})-\bar{v}(A)$.

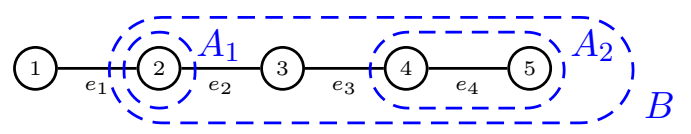

Figure 2: A counterexample to inheritance of convexity with $w_{1}=w_{2}<$ $w_{3}=w_{4}$.

Remark 2. We could also use Theorem 3 to prove that there is no inheritance of convexity in the preceding example.

\section{Relation between the Myerson restricted game and the $\mathcal{P}_{\min }$-restricted game}

Let $G=(N, E)$ be a given graph. We denote by $\mathcal{P}_{M}$ the correspondence on $N$ which assigns to each subset $A \subseteq N$ the partition $\mathcal{P}_{M}(A)$ of $A$ into its connected components. We build from $G$ a weighted graph $G^{\prime}=\left(N^{\prime}, E^{\prime}, w\right)$ as follows. We add a new vertex $s$ to $G$ and an edge joining $s$ to $i$ for each $i \in N$. Hence $N^{\prime}=N \cup\{s\}$ and $E^{\prime}=E \cup\{\{s, i\}, i \in N\}$. For the weight function, we set $w(e)=1$ if $e \in E$ and $w(e)=\frac{1}{2}$ if $e \in E^{\prime} \backslash E$ (we can take any value in $] 0,1\left[\right.$ instead of $\frac{1}{2}$ ). For example, if $G$ is a cycle on five vertices, then $G^{\prime}$ corresponds to the graph represented in Figure 3. We consider the

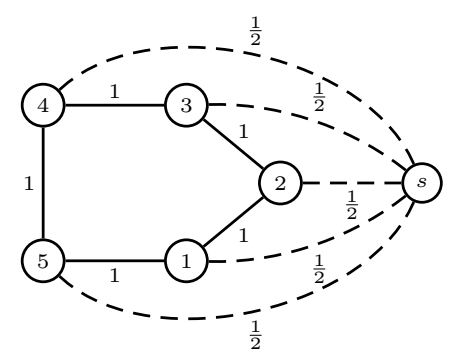

Figure 3: Weighted graph $G^{\prime}$ built from $G$. 
correspondence $\mathcal{P}_{\min }$ on $N^{\prime}$. Let us consider a subset $A^{\prime}$ in $N^{\prime}$. If $A^{\prime} \subseteq N$, then $\mathcal{P}_{\min }\left(A^{\prime}\right)$ is the singleton partition of $A^{\prime}$. Otherwise $A^{\prime}=A \cup\{s\}$ with $A=A^{\prime} \cap N$ and $\mathcal{P}_{\min }\left(A^{\prime}\right)=\left\{\mathcal{P}_{M}(A),\{s\}\right\}$. Let $\left(N^{\prime}, v\right)$ be a zero-normalized game on $N^{\prime}$. Then, the $\mathcal{P}_{\text {min }}$-restricted game $\left(N^{\prime}, \bar{v}\right)$ associated with $G^{\prime}$ satisfies for all $A \subseteq N$ :

$$
\bar{v}(A)=0
$$

and

$$
\bar{v}(A \cup\{s\})=\sum_{F \in \mathcal{P}_{M}(A)} v(F)=v^{M}(A) .
$$

Let us observe that, by (9) and (10), the $\mathcal{P}_{\text {min }}$-restricted game $\left(N^{\prime}, \bar{v}\right)$ only depends on the restriction of $v$ to $N$, and the restriction of $\left(N^{\prime}, \bar{v}\right)$ to the set of coalitions containing $s$ corresponds to the Myerson restricted game on $N$.

Theorem 5. Let $G=(N, E)$ be a graph and $G^{\prime}=\left(N^{\prime}, E^{\prime}, w\right)$ be the weighted connected graph built from $G$ by the preceding procedure. Let us consider a zero-normalized game $\left(N^{\prime}, v\right)$. Let $\mathcal{F}$ be the family of connected subsets of $N^{\prime}$. Then the $\mathcal{P}_{\min }$-restricted game $\left(N^{\prime}, \bar{v}\right)$ is $\mathcal{F}$-convex if and only if $\left(N, v^{M}\right)$ is convex.

Proof. Let us assume that $\left(N^{\prime}, \bar{v}\right)$ is $\mathcal{F}$-convex. Then, for any subsets $A, B \subseteq$ $N$, we have:

$$
\bar{v}(A \cup B \cup\{s\})+\bar{v}(A \cap B \cup\{s\}) \geq \bar{v}(A \cup\{s\})+\bar{v}(B \cup\{s\}) .
$$

(10) implies that (11) is equivalent to:

$$
v^{M}(A \cup B)+v^{M}(A \cap B) \geq v^{M}(A)+v^{M}(B) .
$$

Hence $\mathcal{F}$-convexity of $\left(N^{\prime}, \bar{v}\right)$ implies convexity of $\left(N, v^{M}\right)$.

Let us now assume that $\left(N, v^{M}\right)$ is convex. Then (12) and therefore (11) are satisfied for any subsets $A, B \subseteq N$. As $\left(N, v^{M}\right)$ is convex it is also superadditive and therefore for any subsets $A, B \subseteq N$, we have:

$$
v^{M}(A \cup B) \geq v^{M}(A),
$$

which by (9) and (10) is equivalent to:

$$
\bar{v}(A \cup B \cup\{s\})+\bar{v}(A \cap B) \geq \bar{v}(A \cup\{s\})+\bar{v}(B) .
$$

By symmetry we also have $\bar{v}(A \cup B \cup\{s\})+\bar{v}(A \cap B) \geq \bar{v}(A)+\bar{v}(B \cup\{s\})$. And $\bar{v}(A \cup B)+\bar{v}(A \cap B) \geq \bar{v}(A)+\bar{v}(B)$ is obviously satisfied for all $A, B \subseteq N$. Hence $\left(N^{\prime}, \bar{v}\right)$ is $\mathcal{F}$-convex. 
Corollary 6. Let $G=(N, E)$ be a graph and $G^{\prime}=\left(N^{\prime}, E^{\prime}, w\right)$ be the weighted connected graph obtained from $G$ by the preceding procedure. Let us consider a zero-normalized game $\left(N^{\prime}, v\right)$. Let $\mathcal{F}$ be the family of connected subsets of $N^{\prime}$. Inheritance of $\mathcal{F}$-convexity from $\left(N^{\prime}, v\right)$ to the $\mathcal{P}_{\min }$-restricted game $\left(N^{\prime}, \bar{v}\right)$ is equivalent to inheritance of convexity from $(N, v)$ to $\left(N, v^{M}\right)$.

Note that, although these results establish a link between $\mathcal{F}$-convexity for $\mathcal{P}_{\min }$ and convexity for Myerson's correspondence, they are only valid for the specific graph $G^{\prime}$ built from the arbitrary graph $G$ associated with Myerson restricted game. They directly follow from the fact that we can get Myerson restricted game as a restriction of the $\mathcal{P}_{\min }$-restricted game with the specific construction described at the beginning of this section. The reverse problem of obtaining the $\mathcal{P}_{\min }$-restricted game as a restriction or as a special case of Myerson restricted game seems difficult if not impossible. For any weighted graph $G^{\prime}$ we have to be able to build an auxiliary unweighted graph $G$ such that connectedness is in a certain sense sufficient to find the structure and the distribution of edge-weights of the initial graph. For instance, if $G^{\prime}$ is a weighted tree then $G$ cannot be a subgraph of $G^{\prime}$ otherwise $G$ is also a tree and there is always inheritance of convexity from the underlying game to Myerson restricted game. Hence, we have to construct $G$ from $G^{\prime}$ by adding specific cycles. These cycles have to be complete or not complete according to the distribution of edge-weights. The difficulty of such a construction of $G$ from $G^{\prime}$ already appears in the simplest cases where $G^{\prime}$ is a star or a path. It strongly suggests that we cannot obtain similar results for an arbitrary graph $G^{\prime}$ associated with the $\mathcal{P}_{\min }$-restricted game by easily exhibiting a particular graph $G$.

\section{$5 \quad$ Inheritance of $\mathcal{F}$-convexity for $\mathcal{P}_{\text {min }}$}

Let $G=(N, E, w)$ be a weighted graph. We denote by $w_{j}$ the weight of an edge $e_{j}$ in $E$. Let $\mathcal{F}$ be the family of connected subsets of $N$. We establish in this part necessary and sufficient conditions on the weight vector $w$ for the inheritance of $\mathcal{F}$-convexity from the original game $(N, v)$ to the $\mathcal{P}_{\text {min }^{-}}$ restricted game $(N, \bar{v})$.

\subsection{Necessary conditions on edge-weights}

Throughout this section, $\mathcal{F}$ is the family of connected subsets of $N$. We first establish that there exists a necessary condition associated with every subgraph of $G$ corresponding to a star. A star $S_{k}$ corresponds to a tree with one internal vertex and $k$ leaves. We present the result for stars with three leaves. The generalization to stars of greater size is immediate. We consider a star $S_{3}$ with vertices $\{1,2,3,4\}$ and edges $e_{1}=\{1,2\}, e_{2}=\{1,3\}$ and 
$e_{3}=\{1,4\}$. The following necessary condition already appeared in (Grabisch and Skoda, 2012) but was limited to induced stars ${ }^{5}$. We extend it to all stars of a given graph.

Star Condition. For every star of type $S_{3}$ of $G$, the edge-weights $w_{1}, w_{2}, w_{3}$ satisfy, after renumbering the edges if necessary:

$$
w_{1} \leq w_{2}=w_{3}
$$

Proposition 7. Let $G=(N, E, w)$ be a weighted graph. If for every unanimity game $\left(N, u_{S}\right)$, the $\mathcal{P}_{\min }$-restricted game $\left(N, \overline{u_{S}}\right)$ is $\mathcal{F}$-convex, then the Star Condition is satisfied.

Proof. We have to prove that we cannot have two edge-weights strictly smaller than a third one. By contradiction let us assume we have $w_{1} \leq$ $w_{2}<w_{3}$, after renumbering if necessary. Let us consider the situation of

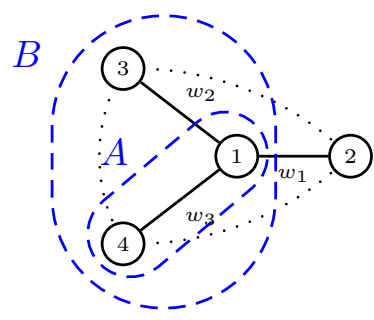

Figure 4: Star of type $S_{3}$ with $w_{1} \leq w_{2}<w_{3}$.

Figure 4 where $A=\{1,4\}, B=\{1,3,4\}$, and $i=2$. Let us observe that, as the star is not necessarily induced, edges $\{2,3\},\{3,4\}$, and $\{2,4\}$ may exist. By deleting the edges of minimum weight we obtain $\mathcal{P}_{\min }(A)=\{\{1\},\{4\}\}$, and $\mathcal{P}_{\min }(B)=\{A,\{3\}\}$ or $\mathcal{P}_{\min }(B)=\{B\}$. If $\mathcal{P}_{\min }(A \cup\{i\})=\{A,\{i\}\}$ we have $A \in \mathcal{P}_{\text {min }}(A \cup\{i\})$ but $\mathcal{P}_{\min }(B)_{\mid A}=A \neq \mathcal{P}_{\min }(A)_{\mid A}$ and it contradicts Theorem 3. Now if $\mathcal{P}_{\min }(A \cup\{i\})=\{A \cup\{i\}\}$ we still get a contradiction since we have $\mathcal{P}_{\min }(B)_{\mid A \cup\{i\}}=A \neq \mathcal{P}_{\min }(A)_{\mid A \cup\{i\}}$.

For a weighted graph $G=(N, E, w)$, we denote by $M$ the maximum edge-weight, i.e., $M=\max _{e \in E} w(e)$, and by $E^{M}$ the set of edges of maximum weight in $G$, i.e., $E^{M}=\{e \in E \mid w(e)=M\}$. A subset $A \subseteq N$ is $M$-maximal if $A$ is a maximal subset w.r.t. inclusion such that $A$ is connected, and $E(A) \subseteq E^{M}$. For a vertex $x \in V$, we denote by $N(x)$ its neighborhood (i.e., the set of vertices adjacent to $x$ ). The neighborhood of a subset $A$ of $N$, is defined by $N(A):=\cup_{x \in A} N(x)$. We say that a vertex $i \in N(A) \backslash A$ is a neighbor of the set $A$, and we denote by $E(A, i)$ the set of edges joining $i$ to $A$ in $G$.

\footnotetext{
${ }^{5}$ An induced star in $G=(N, E)$ is an induced graph $G_{A}=(A, E(A))$ with $A \subseteq N$ and $|A| \geq 4$ corresponding to a star.
} 
Lemma 8. Let $G=(N, E, w)$ be an arbitrary weighted graph and let us consider an $M$-maximal subset $A$ of $N$. For all $i \in N(A) \backslash A$, we have $\mathcal{P}_{\min }(A \cup\{i\})_{\mid A}=\{A\}$.

Proof. We cannot have $w(e)=M$ for all $e \in E(A, i)$ otherwise $A$ is not $M$-maximal. Therefore there exists at least one edge $e=\{i, j\} \in E(A, i)$ such that $w(e)<M$. If all edges in $E(A, i)$ have the same weight $w(e)$, then $\mathcal{P}_{\text {min }}(A \cup\{i\})=\{A,\{i\}\}$. Otherwise $\mathcal{P}_{\min }(A \cup\{i\})=\{A \cup\{i\}\}$.

Lemma 9. Let $G=(N, E, w)$ be an arbitrary weighted graph. If for every unanimity game $\left(N, u_{S}\right)$, the $\mathcal{P}_{\min }$-restricted game $\left(N, \overline{u_{S}}\right)$ is $\mathcal{F}$-convex, then an $M$-maximal subset $A$ of $N$ has at most one neighbor in $N \backslash A$.

Proof. Let us assume $A$ is $M$-maximal and has two distinct neighbors denoted by 1 and 2 in $N$. Let us consider $B=A \cup\{2\}$ and $i=1$. Applying Lemma 8, we have $\mathcal{P}_{\min }(A \cup\{i\})_{\mid A}=\{A\}$ (resp. $\left.\mathcal{P}_{\min }(B)_{\mid A}=\{A\}\right)$. Then $\mathcal{P}_{\min }(B)_{\mid A}=\{A\} \neq \mathcal{P}_{\min }(A)_{\mid A}$ and it contradicts Theorem 3 .

Let $\gamma=\left(e_{1}, e_{2} \ldots, e_{m}\right)$ be an elementary path of $G$ (i.e., a path with no repeated vertices) with $e_{i}=\{i, i+1\}$ for $1 \leq i \leq m$. We now establish that we have a property of convexity on the edge-weights along every elementary path in $G$.

Path Condition. For every elementary path $\gamma=\left(1, e_{1}, 2, e_{2}, 3, \ldots, m\right.$, $\left.e_{m}, m+1\right)$ in $G$ and for all $i, j, k$ such that $1 \leq i<j<k \leq m$, the edge-weights satisfy:

$$
w_{j} \leq \max \left(w_{i}, w_{k}\right)
$$

Proposition 10. Let $G=(N, E, w)$ be a weighted graph. If for every unanimity game $\left(N, u_{S}\right)$, the $\mathcal{P}_{\text {min }^{-} \text {-restricted game }}\left(N, \overline{u_{S}}\right)$ is $\mathcal{F}$-convex, then the Path Condition is satisfied.

Proof. It is sufficient to prove that we have:

$$
w_{j} \leq \max \left(w_{1}, w_{m}\right), \forall j, 1 \leq j \leq m .
$$

Actually, we can do the same reasoning with $w_{i}, w_{k}$ by considering the restriction of $\gamma$ between $i$ and $k+1$. Let $G^{\prime}=\left(N^{\prime}, E^{\prime}, w\right)$ be the subgraph of $G$ induced by the vertices of $\gamma$. Let us consider an $M$-maximal subset $A$ in $G^{\prime}$. Then $A$ contains the end-vertices of $e_{1}$ or $e_{m}$ otherwise it has two neighbors in $N^{\prime} \backslash A$, contradicting Lemma 9. Therefore, $w_{1}=M$ or $w_{m}=M$ and (15) is satisfied.

Remark 3. Moreover we have proved that $\max _{e \in E^{\prime}} w(e)=\max _{e \in \gamma} w(e)=$ $\max \left(w_{1}, w_{m}\right)$. 
We can also obtain necessary conditions for inheritance of convexity in the case of a simple cycle in $G$ (i.e., a cycle with no repeated vertices or edges except for the start and end vertex). For a given cycle $C$, we denote by $E(C)$ the set of edges in $E$ having their end vertices in $\mathrm{C}$.

Cycle Condition. For every simple cycle of $G, C=$ $\left(1, e_{1}, 2, e_{2}, \ldots, m, e_{m}, 1\right)$ with $m \geq 3$, the edge-weights satisfy, after renumbering the edges if necessary:

$$
w_{1} \leq w_{2} \leq w_{3}=\cdots=w_{m}=M
$$

where $M=\max _{e \in E(C)} w(e)$. Moreover $w(e)=w_{2}$ for all chord incident to 2 , and $w(e)=M$ for all chord non incident to 2 .

Proposition 11. Let $G=(N, E, w)$ be a weighted graph. If for every unanimity game $\left(N, u_{S}\right)$, the $\mathcal{P}_{\min }$-restricted game $\left(N, \overline{u_{S}}\right)$ is $\mathcal{F}$-convex, then the Cycle Condition is satisfied.

Proof. If $|E(C)|=3$ then the results are obviously satisfied. Let us assume $|E(C)| \geq 4$. Let us denote by $G^{\prime}=\left(N^{\prime}, E^{\prime}, w\right)$ the subgraph of $G$ induced by the vertices of $C$. Let $A$ be an $M$-maximal subset of $G^{\prime}$. Lemma 9 implies $\left|N^{\prime} \backslash A\right| \leq 1$, therefore (16) is obviously satisfied, after renumbering the edges if necessary. Moreover, as $w(e)=M$ for all $e \in E(A)$, we have $w(e)=M$ for all chord $e$ non incident to 2 . Let us now consider a chord $e=\{2, j\}$ incident to 2 . Then, Proposition 7 applied to the star defined by $\left\{e_{1}, e_{2}, e\right\}$ implies $w_{1} \leq w_{2}=w(e)$ or $w(e) \leq w_{1}=w_{2}$. By contradiction, let us assume $w(e)<w_{1}=w_{2}$. Using Star condition, we have $w\left(e^{\prime}\right)=w_{1}=w_{2}$ for all other chords $e^{\prime}=\{2, k\}$ incident to 2. If such a chord $e^{\prime}$ exists, we can consider a cycle smaller than $C$ by replacing the vertex 1 or 2 by $k$. Hence we can assume that $e$ is a unique chord incident to 2 . Let us consider $A=V(C) \backslash\{1,3\}, B=V(C) \backslash\{1\}$, and $i=1$ as represented in Figure 5. Then $\mathcal{P}_{\min }(A \cup\{i\})=\{A \cup\{i\}\}, \mathcal{P}_{\text {min }}(B)=\{B\}$, and $\mathcal{P}_{\min }(A)=$ $\{\{2\}, A \backslash\{2\}\}$. Therefore $\mathcal{P}_{\min }(B)_{\mid A \cup\{i\}}=\{A\} \neq \mathcal{P}_{\min }(A)_{\mid A \cup\{i\}}$ and it contradicts Theorem 3 .

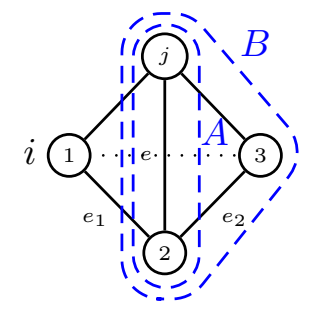

Figure 5: Cycle $C$ with $w(e)<w_{1}=w_{2}$.

Let us consider a weighted graph $G=(N, E, w)$ and a cycle $C$ in $G$. If 
$e \in E(C)$ is a chord of $C$ such that $w(e)=\max _{e \in E(C)} w(e)$, we say that $e$ is a maximum weight chord.

Pan Condition. For all connected subgraphs corresponding to the union of a simple cycle $C=\left\{e_{1}, e_{2}, \ldots, e_{m}\right\}$ with $m \geq 3$, and an elementary path $P$ such that there is an edge $e$ in $P$ with $w(e) \leq \min _{1 \leq k \leq m} w_{k}$ and $|V(C) \cap V(P)|=1$, the edge-weights satisfy:

$$
\begin{aligned}
\text { either } & w_{1}=w_{2}=w_{3}=\cdots=w_{m}=M, \\
\text { or } & w_{1}=w_{2}<w_{3}=\cdots=w_{m}=M,
\end{aligned}
$$

where $M=\max _{e \in E(C)} w(e)$. In this last case $V(C) \cap V(P)=\{2\}$ and if moreover $w(e)<w_{1}$ then $\{1,3\}$ is a maximum weight chord of $C$.

Proposition 12. Let $G=(N, E, w)$ be a weighted graph. If for every unanimity game $\left(N, u_{S}\right)$, the $\mathcal{P}_{\min }$-restricted game $\left(N, \overline{u_{S}}\right)$ is $\mathcal{F}$-convex, then the Pan Condition is satisfied.

Proof. Let us consider $C=\left\{1, e_{1}, 2, e_{2}, 3, \ldots, m, e_{m}, 1\right\}$, and $P=\left\{j, e_{m+1}\right.$, $\left.m+1, e_{m+2}, m+2, \ldots, e_{m+r}, m+r\right\}$ with $j \in\{1, \ldots, m\}$, as represented in Figure 6. We can assume w.l.o.g. that $e=e_{m+r}$ (restricting $P$ if necessary) and that $w_{m+j}>w_{m+r}=w(e)$ for all $1 \leq j \leq r-1$. Applying Proposition 11

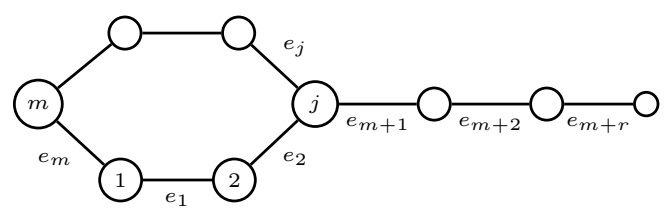

Figure 6: Pan formed by the union of $C$ and $P$.

to the cycle $C$, we have after renumbering the edges if necessary:

$$
w_{1} \leq w_{2} \leq w_{3}=\cdots=w_{m}=M .
$$

a) Let us first assume $3 \leq j \leq m$. Applying Proposition 10 to the path $\left\{2, e_{1}, 1, e_{m}, m, \ldots, j+1, e_{j}, j, e_{m+1}, m+1, \ldots, m+r-1, e_{m+r}, m+r\right\}$, we have $w_{j} \leq \max \left(w_{1}, w(e)\right)=w_{1}$. Then (19) implies (17).

b) Let us now assume $j \in\{1,2\}$. If $r=1$ then $w_{m+1}=w(e) \leq w_{1}$. Otherwise, applying Proposition 10 to the path $\left\{e_{1}, e_{m+1} \ldots, e_{m+r}\right\}$, we have $w_{m+1} \leq \max \left(w_{1}, w(e)\right)=w_{1}$.

If $j=1$, Proposition 7 applied to the star defined by $\left\{e_{1}, e_{m}, e_{m+1}\right\}$, implies $w_{m+1} \leq w_{1}=w_{m}$. Hence (19) still implies (17).

If $j=2$, Proposition 7 applied to the star defined by $\left\{e_{1}, e_{2}, e_{m+1}\right\}$, implies $w_{m+1} \leq w_{1}=w_{2}$. If $w_{1}=w_{2}=M$, then (17) is satisfied. Otherwise, we have $w_{1}=w_{2}<M$ and therefore (18) is satisfied. In this last case, let us assume by contradiction that $\{1,3\} \notin E(C)$. Proposition 11 implies 
$w(e)=M$ (resp. $w(e)=w_{2}$ ) for any chord $e$ of $C$ non incident (resp. incident) to 2. Therefore we can assume w.l.o.g. that $C$ has no maximum weight chord (otherwise we can replace $C$ by a smaller cycle which still contains the vertices 1,2,3). Let us consider $i \in V(C) \backslash\{1,2,3\}, A=$ $V(C) \backslash\{i\}$ and $B=A \cup V(P)$ as represented in Figure 7. Then $\mathcal{P}_{\min }(A)=$

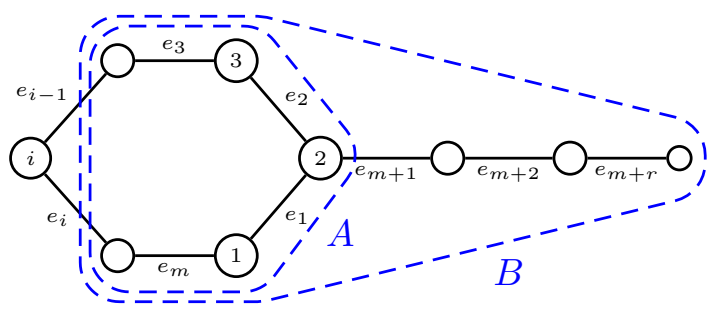

Figure 7: $w_{m+r}<w_{1}=w_{2}<w_{3}=\cdots=w_{m}=M$.

$\{\{2\},\{3,4, \ldots, i-1\},\{i+1, \ldots, m, 1\}\}, \mathcal{P}_{\min }(A \cup\{i\})=\{A \cup\{i\} \backslash\{2\},\{2\}\}$, and $\mathcal{P}_{\min }(B)=\{B \backslash\{m+r\},\{m+r\}\}$ or $\mathcal{P}_{\min }(B)=\{B\}$ (this last case can occur if there exists an edge $e^{\prime}$ in $G_{B}$ with $w\left(e^{\prime}\right)<w(e)$ or with $m+r$ as an end-vertex and $\left.w\left(e^{\prime}\right)>w(e)\right)$. Therefore $A^{\prime}:=A \cup\{i\} \backslash\{2\} \in \mathcal{P}_{\min }(A \cup\{i\})$, but $\mathcal{P}_{\min }(B)_{\mid A^{\prime}}=\{A \backslash\{2\}\} \neq \mathcal{P}_{\min }(A)_{\mid A^{\prime}}$ and it contradicts Theorem 3 .

Let $G=(N, E, w)$ be a graph. We say that two cycles are adjacent if they have at least one common edge. Let us consider a cycle $C=$ $\left(1, e_{1}, 2, e_{2}, \ldots, m, e_{m}, 1\right)$ such that $w_{1} \leq w_{2} \leq w_{3}=\ldots=w_{m}$. If $w_{1}<w_{3}$ (resp. $w_{2}<w_{3}$ ), we say that $e_{1}$ (resp. $e_{2}$ ) is a non-maximum weight edge of $C$.

Lemma 13. Let $G=(N, E, w)$ be a weighted graph satisfying the Star and Cycle conditions. Then for all pairs $\left(C, C^{\prime}\right)$ of adjacent simple cycles in $G$, we have:

$$
M=\max _{e \in E(C)} w(e)=\max _{e \in E\left(C^{\prime}\right)} w(e)=M^{\prime} .
$$

Proof. Let us consider two adjacent cycles $C$ and $C^{\prime}$ with $M<M^{\prime}$. There is at least one edge $e_{1}$ common to $C$ and $C^{\prime}$. Then, we have $w_{1} \leq M<M^{\prime}$ and therefore $e_{1}$ is a non-maximum weight edge in $C^{\prime}$. The Cycle condition implies that there are at most two non-maximum weight edges in $C^{\prime}$ (cf. Proposition 11). Therefore there exists an edge $e_{2}^{\prime}$ in $C^{\prime}$ adjacent to $e_{1}$ with $w_{2}^{\prime}=M^{\prime}$. As $M^{\prime}>M, e_{2}^{\prime}$ is not an edge of $C$. Let $e_{2}$ be the edge of $C$ adjacent to $e_{1}$ and $e_{2}^{\prime}$. Then, we have $w_{2} \leq M<M^{\prime}$ but it contradicts the Star condition applied to $\left\{e_{1}, e_{2}, e_{2}^{\prime}\right\}$ (two edge-weights are strictly smaller than $\left.w_{2}^{\prime}\right)$. 
Adjacent Cycles Condition. For all pairs $\left(C, C^{\prime}\right)$ of adjacent simple cycles in $G$ such that:

1. $V(C) \backslash V\left(C^{\prime}\right) \neq \emptyset$ and $V\left(C^{\prime}\right) \backslash V(C) \neq \emptyset$,

2. $C$ has at most one non-maximum weight chord,

3. $C$ and $C^{\prime}$ have no maximum weight chord,

4. $C$ and $C^{\prime}$ have no common chord,

then $C$ and $C^{\prime}$ cannot have two common non-maximum weight edges. Moreover $C$ and $C^{\prime}$ have a unique common non-maximum weight edge $e_{1}$ if and only if there are non-maximum weight edges $e_{2} \in E(C) \backslash E\left(C^{\prime}\right)$ and $e_{2}^{\prime} \in E\left(C^{\prime}\right) \backslash E(C)$ such that $e_{1}, e_{2}, e_{2}^{\prime}$ are adjacent and:

- $w_{1}=w_{2}=w_{2}^{\prime}$ if $|E(C)| \geq 4$ and $\left|E\left(C^{\prime}\right)\right| \geq 4$.

- $w_{1}=w_{2} \geq w_{2}^{\prime}$ or $w_{1}=w_{2}^{\prime} \geq w_{2}$ if $|E(C)|=3$ or $\left|E\left(C^{\prime}\right)\right|=3$.

Proposition 14. Let $G=(N, E, w)$ be a weighted graph. Let us assume that for every unanimity game $\left(N, u_{S}\right)$, the $\mathcal{P}_{\min }$-restricted game $\left(N, \overline{u_{S}}\right)$ is $\mathcal{F}$-convex. Then the Adjacent Cycles Condition is satisfied.

Proof. Let us consider two adjacent cycles $C=\left\{1, e_{1}, 2, e_{2}, \ldots, m, e_{m}, 1\right\}$ and $C^{\prime}=\left\{1^{\prime}, e_{1}^{\prime}, 2^{\prime}, e_{2}^{\prime}, \ldots, p^{\prime}, e_{p}^{\prime}, 1^{\prime}\right\}$. By Lemma 13 , we have $M=\max _{e \in E(C)}$ $w(e)=\max _{e \in E\left(C^{\prime}\right)} w(e)=M^{\prime}$. Proposition 11 implies that there are at most two non-maximum weight edges in $E(C) \cap E\left(C^{\prime}\right)$.

A) Let us first assume that there are exactly two common non-maximum weight edges $e_{1}, e_{2}$. Therefore we can assume $e_{1}=e_{1}^{\prime}, e_{2}=e_{2}^{\prime}, j=j^{\prime}$ for $1 \leq j \leq 3$. Of course, $C$ and $C^{\prime}$ may have other common edges or vertices. Hence we have:

$$
w_{1} \leq w_{2}<M=\max _{e \in E(C)} w(e)=\max _{e \in E\left(C^{\prime}\right)} w(e)=M^{\prime} .
$$

Proposition 11 also implies that all chords of $C$ and $C^{\prime}$ are incident to 2 and have weight $w_{2}$. By assumption, $C$ has at most one non-maximum weight chord in $E(C) \backslash E\left(C^{\prime}\right)$. If $C$ has one non-maximum weight chord $e$ in $E(C) \backslash E\left(C^{\prime}\right)$, then $e=\{2, i\}$ with $i \in V(C) \backslash V\left(C^{\prime}\right)$. Let us define $A=V(C) \backslash\{i\}, B=A \cup V\left(C^{\prime}\right)$ as represented in Figure 8 with $m=p=6$. If $C$ has no chord, then we choose $i \in V(C) \backslash V\left(C^{\prime}\right)$ arbitrarily. We now consider two cases.

Case 1 If $w_{1}<w_{2}$, then $\mathcal{P}_{\min }(A)=\{\{2,3, \ldots, i-1\},\{i+1, \ldots, m, 1\}\}$, $\mathcal{P}_{\min }(A \cup\{i\})=\{A \cup\{i\}\}$, and $\mathcal{P}_{\min }(B)=\{B\}$. Therefore $A^{\prime}:=A \cup\{i\} \in$ $\mathcal{P}_{\min }(A \cup\{i\})$, but $\mathcal{P}_{\min }(B)_{\mid A^{\prime}}=\{A\} \neq \mathcal{P}_{\min }(A)_{\mid A^{\prime}}$ and it contradicts Theorem 3 . 


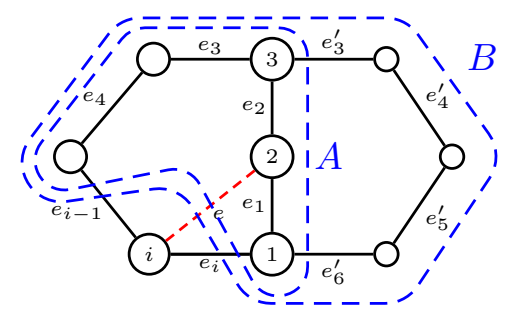

Figure 8: $C$ and $C^{\prime}$ with two common non-maximum weight edges.

Case 2 Now if $w_{1}=w_{2}$, we have $\mathcal{P}_{\min }(A)=\{\{2\},\{3,4, \ldots, i-1\},\{i+$ $1, \ldots, m, 1\}\}, \mathcal{P}_{\min }(A \cup\{i\})=\{A \cup\{i\} \backslash\{2\},\{2\}\}$, and $\mathcal{P}_{\min }(B)=\{B \backslash$ $\{2\},\{2\}\}$ (as $V\left(C^{\prime}\right) \backslash V(C) \neq \emptyset$ ). Taking $A^{\prime}=A \cup\{i\} \backslash\{2\}$, we have $\mathcal{P}_{\text {min }}(B)_{\mid A^{\prime}}=\{A \backslash\{2\}\} \neq \mathcal{P}_{\min }(A)_{\mid A^{\prime}}$, and it still contradicts Theorem 3 .

B) Let us now assume that there is exactly one common non-maximum weight edge $e_{1}$. Therefore we can assume $e_{1}=e_{1}^{\prime}, j=j^{\prime}$ for $1 \leq j \leq 2$, and we have:

$$
w_{1}<M=\max _{e \in E(C)} w(e)=\max _{e \in E\left(C^{\prime}\right)} w(e)=M^{\prime} .
$$

If the edges in $C$ (resp. $C^{\prime}$ ) adjacent to $e_{1}$ are maximum weight edges then Proposition 11 applied to $C$ (resp. $C^{\prime}$ ) implies $w(e)=M$ for all $e \in E(C) \backslash\left\{e_{1}\right\}$ (resp. $e \in E\left(C^{\prime}\right) \backslash\left\{e_{1}\right\}$ ). Therefore $C$ and $C^{\prime}$ have no chord. We choose arbitrarily a vertex $i \in V(C) \backslash V\left(C^{\prime}\right)$. Then taking $A=V(C) \backslash\{i\}$, and $B=A \cup V\left(C^{\prime}\right)$, we get the same contradiction as in Case 1. If there is a non-maximum weight edge $e$ in $C$ or $C^{\prime}$ adjacent to $e_{1}$, then we can assume w.l.o.g. $e=e_{2}=\{2,3\} \in C$. Let us consider $e_{2}^{\prime}=\left\{2,3^{\prime}\right\} \in C^{\prime}$. We necessarily have $3 \neq 3^{\prime}$ otherwise $e_{2}^{\prime}=e_{2}$ and $e_{1}, e_{2}$ would be two common non-maximum weight edges, a contradiction. If $e_{2}^{\prime}$ is a maximum weight edge, then it contradicts Proposition 7 applied to the star defined by $\left\{e_{1}, e_{2}, e_{2}^{\prime}\right\}$. Therefore $e_{2}^{\prime}$ is a non-maximum weight edge. Proposition 7 applied to the star defined by $\left\{e_{1}, e_{2}, e_{2}^{\prime}\right\}$ implies $w_{1} \leq w_{2}=w_{2}^{\prime}$ or $w_{2}^{\prime} \leq w_{1}=w_{2}$ or $w_{2} \leq w_{1}=w_{2}^{\prime}$. Let us first assume $w_{1}<w_{2}=w_{2}^{\prime}$. Then we can establish the same contradiction as in Case 1. Hence, Proposition 14 is satisfied if $E(C)=3$ or $E\left(C^{\prime}\right)=3$. Let us now assume $w_{2}^{\prime}<w_{1}=w_{2}$. If $|E(C)| \geq 4$, then, as $C$ has no chord, it contradicts Proposition 12 applied to the pan defined by $C$ and $e_{2}^{\prime}$ and represented in Figure 9. By symmetry

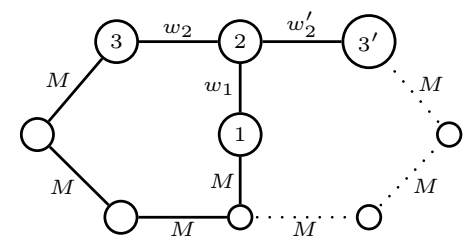

Figure 9: $w_{2}^{\prime}<w_{1}=w_{2}<M$ contradicts the Pan condition. 
the case $w_{2}<w_{1}=w_{2}^{\prime}$ is also impossible if $\left|E\left(C^{\prime}\right)\right| \geq 4$. Finally, if $e_{1}, e_{2}$ and $e_{2}^{\prime}$ satisfies the conditions of the proposition, then $e_{1}$ is the unique common non-maximum weight edge as each cycle cannot have more than two nonmaximum weight edges.

Remark 4. We consider simple cycles in Proposition 14 to avoid situations similar to the one represented in Figure 10. In this case we cannot establish

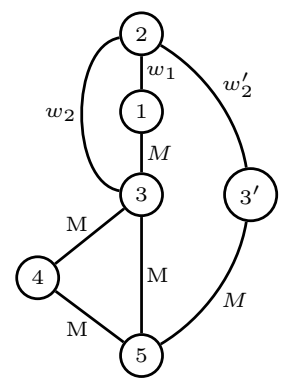

Figure 10: $C=\left\{1, e_{1}, 2, e_{2}, 3, e_{3}, 4, e_{4}, 5, e_{5}, 3, e_{6}, 1\right\}, \quad C^{\prime}=$ $\left\{1, e_{1}, 2, e_{2}^{\prime}, 3^{\prime}, e_{3}^{\prime}, 5, e_{5}, 3, e_{6}, 1\right\}$ with $w_{1}<w_{2}=w_{2}^{\prime}<M$ but $C$ is not a simple cycle.

the same contradiction as in the proof of Proposition 14 even though $V(C) \backslash$ $V\left(C^{\prime}\right) \neq \emptyset$ and $V\left(C^{\prime}\right) \backslash V(C) \neq \emptyset$.

\subsection{Sufficient conditions}

Let $\mathcal{F}$ be the family of connected subsets of $N$. We will now prove that the necessary conditions defined in Section 5.1 are also sufficient for superadditive games. This constitutes the main result of the paper.

Theorem 15. Let $G=(N, E, w)$ be a weighted graph. For every superadditive and $\mathcal{F}$-convex game $(N, v)$, the $\mathcal{P}_{\text {min }}$-restricted game $(N, \bar{v})$ is $\mathcal{F}$-convex if and only if the Path, Star, Cycle, Pan, and Adjacent Cycles conditions are satisfied.

To prove Theorem 15 we first need some useful lemmas. The first one was already established in (Grabisch and Skoda, 2012).

Lemma 16. Let us consider subsets $A, B \subseteq N$ and a partition $\left\{B_{1}, B_{2}, \ldots\right.$, $\left.B_{p}\right\}$ of $B$. If $A, B_{i}$, and $A \cap B_{i} \in \mathcal{F}$, for all $i \in\{1, \ldots, p\}$, then for every $\mathcal{F}$-convex game $(N, v)$ we have:

$$
v(A \cup B)+\sum_{i=1}^{p} v\left(A \cap B_{i}\right) \geq v(A)+\sum_{i=1}^{p} v\left(B_{i}\right) .
$$


Corollary 17. Let us consider a correspondence $\mathcal{P}$ on $N$ and subsets $A \subseteq$ $B \subseteq N$ such that $\mathcal{P}(A)=\mathcal{P}(B)_{\mid A}$. If $A \in \mathcal{F}$ and if all elements of $\mathcal{P}(A)$ and $\mathcal{P}(B)$ are in $\mathcal{F}$, then for every $\mathcal{F}$-convex game $(N, v)$ we have:

$$
v(B)-\bar{v}(B) \geq v(A)-\bar{v}(A) .
$$

Proof. If $\mathcal{P}(B)=\left\{B_{1}, B_{2}, \ldots, B_{p}\right\}$ then $\mathcal{P}(A)=\left\{A \cap B_{1}, A \cap B_{2}, \ldots, A \cap B_{p}\right\}$, and Lemma 16 implies (24).

We say that an edge $e \in E$ is connected to a subset $A \subseteq N$, if there is a path in $G$ joining $e$ to $A$. The following lemma gives simple conditions ensuring $\mathcal{P}_{\min }(A)$ is induced by $\mathcal{P}_{\min }(B)$ for $A \subseteq B$.

Lemma 18. Let $G=(N, E, w)$ be a weighted graph. Let us assume that the edge-weight function $w$ satisfies the Pan condition. Let us consider $A, B \in$ $\mathcal{F}$ such that $A \subseteq B \subseteq N$ and $\sigma(A)=\sigma(B)$. Let us assume that either the subgraph $G_{B}=(B, E(B))$ is cycle-free or there exists an edge $e \in E$ connected to $B$ with $w(e)<\sigma(B)$. Then $\mathcal{P}_{\min }(A)=\mathcal{P}_{\min }(B)_{\mid A}$ and for every $\mathcal{F}$-convex game $(N, v)$ we have:

$$
v(B)-\bar{v}(B) \geq v(A)-\bar{v}(A) .
$$

Proof. Let $B_{k}$ be a component of $\mathcal{P}_{\min }(B)$ such that $A \cap B_{k} \neq \emptyset$. Let $\alpha_{0}$ be a fixed vertex of $A \cap B_{k}$ and $A_{k}$ be the component of $\mathcal{P}_{\min }(A)$ which contains $\alpha_{0}$. We will prove $A \cap B_{k}=A_{k}$. As $\sigma(A)=\sigma(B), \Sigma(A)=E(A) \cap \Sigma(B)$. Let $\alpha_{1}$ be another vertex of $A_{k}$ and $\gamma$ be a path in $A_{k}$ from $\alpha_{0}$ to $\alpha_{1}$. Each edge $e$ of $\gamma$ is in $E(A) \backslash \Sigma(A)$ and therefore also in $E(B) \backslash \Sigma(B)$. Hence $\gamma$ is also a path from $\alpha_{0}$ to $\alpha_{1}$ in $B$ and therefore $\alpha_{1} \in B_{k}$. That is:

$$
A_{k} \subseteq A \cap B_{k} .
$$

Let us assume there is a vertex $\alpha_{1}$ in $A \cap B_{k} \backslash A_{k}$. As $A$ (resp. $B_{k}$ ) is connected, there exists a path $\gamma\left(\right.$ resp. $\gamma^{\prime}$ ) from $\alpha_{0}$ to $\alpha_{1}$ in A (resp. $B_{k}$ ). By definition of $B_{k}$, we have $w(e)>\sigma(A)=\sigma(B)$ for all $e \in \gamma^{\prime}$. As $\alpha_{1} \notin A_{k}$, there exists at least one edge $e^{\prime} \in \gamma$ such that $w\left(e^{\prime}\right)=\sigma(A)=\sigma(B)$. Therefore $\gamma \neq \gamma^{\prime}$ and :

$$
w(e)>w\left(e^{\prime}\right), \forall e \in \gamma^{\prime} .
$$

Hence $\gamma$ and $\gamma^{\prime}$ form a cycle $C$ in $G_{B}$. If $G_{B}$ is cycle-free, we get a contradiction. Otherwise, we can select $\alpha_{0}, \alpha_{1}$ and $\gamma, \gamma^{\prime}$ such that $C$ is a simple cycle without maximum weight chord $\left(\gamma\right.$ and $\gamma^{\prime}$ are paths with minimum number of edges). By assumption, there exists an edge $e^{\prime \prime} \in E$ connected to $B$ such that $w\left(e^{\prime \prime}\right)<\sigma(B)$. Therefore there is a path $P$ linking $e^{\prime \prime}$ to $C$ as represented in Figure 11 and we have $w\left(e^{\prime \prime}\right)<\min _{e \in E(C)} w(e)$. If $|V(C)| \geq 4$ or if $|V(C)|=3$ and $\gamma^{\prime}$ contains two edges then (27) contradicts the Pan condition. Otherwise $\gamma^{\prime}$ is reduced to the edge $e=\left\{\alpha_{0}, \alpha_{1}\right\}$ and therefore (27) implies $\alpha_{1} \in A_{k}$, a contradiction. Finally Corollary 17 implies (25). 


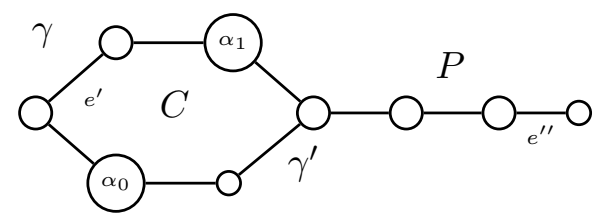

Figure 11: $w(e)>w\left(e^{\prime}\right), \forall e \in \gamma^{\prime}$, and $w\left(e^{\prime \prime}\right)<\min _{e \in E(C)} w(e)$.

Lemma 19. Let $G=(N, E, w)$ be a weighted graph. Let us assume that the edge-weight function $w$ satisfies the Path and Star conditions. For a given $i \in N$, if $A, B \in \mathcal{F}, A \subseteq B \subseteq N \backslash\{i\}$, and $E(A, i) \neq \emptyset$ then either $\sigma(A, i) \geq$ $\sigma(A) \geq \sigma(B)$ or $\sigma(A)=\sigma(B)>\sigma(A, i)$ where $\sigma(A, i)=\min _{e \in E(A, i)} w(e)$.

Proof. As $A \subseteq B$, we have $\sigma(A) \geq \sigma(B)$. Let us assume:

$$
\sigma(A)>\sigma(A, i)
$$

Let $e=\{i, j\}$ be an edge in $E(A, i)$ such that $w(e)=\sigma(A, i)$. As $A$ (resp. $B)$ is connected, there exists an elementary path $\gamma=\left(e_{1}, e_{2}, \ldots, e_{m}\right)$ in $G_{A}$ $\left(\right.$ resp. $\gamma^{\prime}=\left(e_{1}^{\prime}, e_{2}^{\prime}, \ldots, e_{r}^{\prime}\right)$ in $\left.G_{B}\right)$ with $w_{1}=\sigma(A)\left(\right.$ resp. $\left.w_{1}^{\prime}=\sigma(B)\right)$ and such that $j$ is an end-vertex of $e_{m}$ (resp. $e_{r}^{\prime}$ ), as represented in Figure 12. If $\gamma$

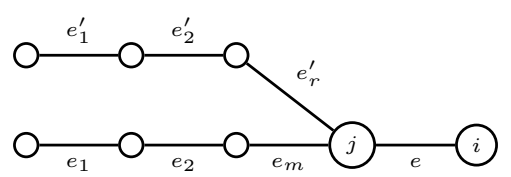

Figure 12: $w(e)=\sigma(A, i), w_{1}=\sigma(A), w_{1}^{\prime}=\sigma(B)$.

is reduced to $e_{1}$ then (28) implies $w_{1}>w(e)$. Otherwise, the Path condition applied to $\gamma \cup\{e\}$ and (28) imply $w_{m} \leq \max \left(w_{1}, w(e)\right)=w_{1}=\sigma(A)$. As $e_{m} \in E(A), w_{m}=\sigma(A)$ and using again (28), we obtain:

$$
w_{m}>w(e) \text {. }
$$

If $e_{r}^{\prime}=e_{m}$, we have $w_{r}^{\prime}=w_{m}=\sigma(A)$. Otherwise, the Star condition applied to $\left\{e_{r}^{\prime}, e_{m}, e\right\}$ and (29) imply again $w_{r}^{\prime}=w_{m}=\sigma(A)$. If $\gamma^{\prime}$ is reduced to $e_{1}^{\prime}$ then $w_{1}^{\prime}=\sigma(A)$ and therefore $\sigma(B)=\sigma(A)$. Otherwise, the Path condition applied to $\gamma^{\prime} \cup\{e\}$ implies $w_{r}^{\prime} \leq \max \left(w_{1}^{\prime}, w(e)\right)$ and therefore:

$$
\sigma(A) \leq \max (\sigma(B), \sigma(A, i)) .
$$

Then (28) and (30) imply $\sigma(A) \leq \sigma(B)$. Therefore $\sigma(A)=\sigma(B)$.

Lemma 20. Let $G=(N, E, w)$ be a weighted graph. Let us assume that the edge-weight function $w$ satisfies the Path, Star, and Cycle conditions. Let us consider $i \in N$ and $A \subseteq B \subseteq N \backslash\{i\}$ with $A, B$, and $A \cup\{i\} \in \mathcal{F}$ and 
such that $\sigma(A, i) \geq \sigma(A) \geq \sigma(B)$. Moreover, let us assume if $\sigma(A)=\sigma(B)$ that the Adjacent Cycles condition is verified, and if $\sigma(A)>\sigma(B)$, that the Pan condition is verified. Let $A_{1}, A_{2}, \ldots, A_{k}$ (resp. $B_{1}, B_{2}, \ldots, B_{l}$ ) be the components of $\mathcal{P}_{\min }(A)$ (resp. $\mathcal{P}_{\min }(B)$ ) connected to $i$ by an edge $e$ in $E(A, i)$ (resp. $E(B, i)$ ) of weight $w(e)>\sigma(A)$. Then we have $k \leq l$ and $A_{j} \subseteq B_{j}$ for all $j, 1 \leq j \leq k$, after renumbering if necessary. Moreover, if $\sigma(A)>\sigma(B)$, then $k=1$.

Proof. As $A \subseteq B$ and as there is inheritance of superadditivity for $\mathcal{P}_{\text {min }}$ (Proposition 4), Theorem 1 implies that each $A_{j}$ with $1 \leq j \leq k$ is a subset of some $B_{p}$ with $1 \leq p \leq l$. Let us prove that if $j_{1} \neq j_{2}$, then $A_{j_{1}} \subseteq B_{j_{1}}$ and $A_{j_{2}} \subseteq B_{j_{2}}$ with $B_{j_{1}} \neq B_{j_{2}}$. By contradiction, let us assume that $A_{1}$ and $A_{2}$ are subsets of $B_{1}$, after renumbering if necessary. There exist edges $\left\{i, j_{1}\right\}$ and $\left\{i, j_{2}\right\}$ with $j_{1} \in A_{1}, j_{2} \in A_{2}$, and weights strictly greater than $\sigma(A)$. As $A$ is connected there exists a path $\gamma$ in $A$ from $j_{1}$ to $j_{2}$. Then $\left\{i, j_{1}\right\} \cup \gamma \cup\left\{j_{2}, i\right\}$ induces a cycle $C_{1}$. Let us select $j_{1}, j_{2}$ and $\gamma$ such that $C_{1}$ is of minimum length. Hence $C_{1}$ has no chord except possibly between $i$ and vertices of $\gamma$. As the components $A_{1}$ and $A_{2}$ in $\mathcal{P}_{\min }(A)$ are obtained by deleting edges of weight $\sigma(A)$ in $E(A)$, there exists at least one edge in $\gamma$ of weight $\sigma(A)$. The Cycle condition applied to $C_{1}$ implies there are two adjacent edges $e_{1}, e_{2}$ with $\sigma(A)=w_{1}<w_{2} \leq M$ or $\sigma(A)=w_{1}=w_{2}<M$ and all other edges in $\gamma$ have weight $M=\max _{e \in E\left(C_{1}\right)} w(e)$ (Note that if $\left|E\left(C_{1}\right)\right|=3$ then we necessarily have $\sigma(A)=w_{1}<w_{2} \leq M$ with $e_{2}$ incident to $i$ ). Hence we can assume $A_{1} \cap C_{1}=\left\{2,3, \ldots, j_{1}=i-1\right\}$ if $\sigma(A)=w_{1}<$ $w_{2} \leq M$, or $A_{1} \cap C_{1}=\left\{3, \ldots, j_{1}=i-1\right\}$ if $\sigma(A)=w_{1}=w_{2}<M$, and $A_{2} \cap C_{1}=\left\{j_{2}=i+1, i+2, \ldots, m, 1\right\}$ as represented in Figure 13. If there is a chord $\{i, j\}$ with $j \in A_{1}$ or $j \in A_{2}$, then it contradicts the minimality of $C_{1}$. Therefore $C_{1}$ has at most one chord $\{i, 2\}$ adjacent to $e_{1}$ and $e_{2}$ (if $\left.w_{1}=w_{2}<M\right)$.

(a)

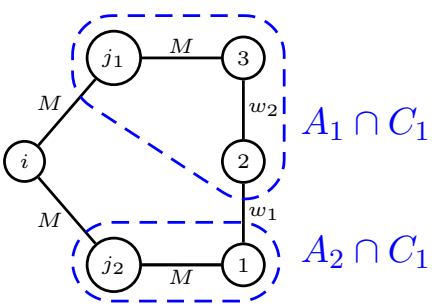

(b)

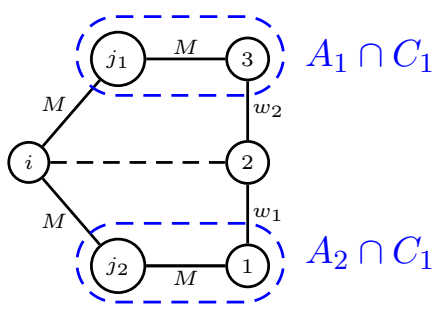

Figure 13: (a) : $w_{1}<w_{2} \leq M,(\mathrm{~b}): w_{1}=w_{2}<M$.

As $A_{1}$ and $A_{2}$ are both subsets of the same component $B_{1} \in \mathcal{P}_{\min }(B)$, there exists a minimum path $\gamma^{\prime}$ in $B_{1}$ linking $1 \in A_{2}$ to $k \in A_{1}$ with $k=2$ if $w_{1}<w_{2}$ and $k=3$ if $w_{1}=w_{2}$.

Let us assume $\sigma(A)=\sigma(B)$. By definition of $B_{1}$ each edge $e^{\prime}$ in $\gamma^{\prime}$ has a weight $w\left(e^{\prime}\right)>\sigma(B)=\sigma(A)$. We get a cycle $C_{2}=\left\{1, e_{1}, 2, \ldots, k\right\} \cup \gamma^{\prime}$. Let 
us remark that if $w_{1}=w_{2}$ then 2 cannot be a vertex of $\gamma^{\prime}$, otherwise there is an edge $e^{\prime}$ in $\gamma^{\prime}$ incident to 2 with $w\left(e^{\prime}\right)>w_{1}=w_{2}$, and it contradicts the Star condition applied to $\left\{e_{1}, e_{2}, e^{\prime}\right\}$ (cf. Figure 14).

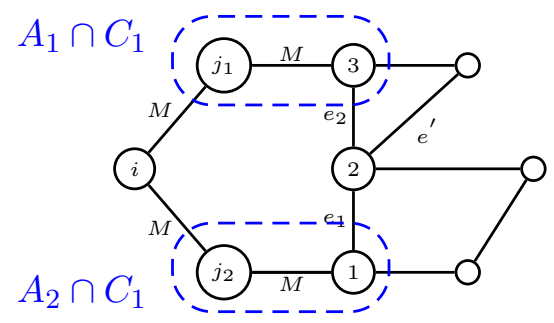

Figure 14: $w_{1}=w_{2}<w\left(e^{\prime}\right)$ contradicts the Star condition.

Let us first assume $k=3$ (i.e., $w_{1}=w_{2}$ ). Then we necessarily have $\left|E\left(C_{1}\right)\right| \geq 4$ and $\left|E\left(C_{2}\right)\right| \geq 4$ (otherwise there is an edge from 1 to 3 with weight strictly greater than $\sigma(A)$, and $A_{1}, A_{2}$ are not disjoint components of $\left.\mathcal{P}_{\min }(A)\right)$. The Cycle condition applied to $C_{2}$ and Lemma 13 imply that all edges $e^{\prime} \in \gamma^{\prime}$ have weight $w\left(e^{\prime}\right)=M$. Any chord in $C_{2}$ non incident to 2 would contradict the minimality of $\gamma^{\prime}$. Moreover, the Cycle condition applied to $C_{1}$ (resp. $C_{2}$ ) implies that any chord $e$ in $C_{1}$ (resp. $C_{2}$ ) incident to 2 satisfies $w(e)=w_{2}$. Therefore $C_{1}$ and $C_{2}$ have no maximum chord. But $C_{1}$ and $C_{2}$ have two common non-maximum weight edges $e_{1}, e_{2}$, contradicting the Adjacent Cycles condition.

Let us now assume $k=2$ (i.e., $w_{1}<w_{2}$ ). Then $C_{1}$ has no chord. $C_{2}$ has no chord, otherwise it contradicts the minimality of $\gamma^{\prime}$. Let $e^{\prime}$ (resp. $e^{\prime \prime}$ ) be the edge of $\gamma^{\prime}$ incident to 1 (resp. 2) as represented in Figure 15. If

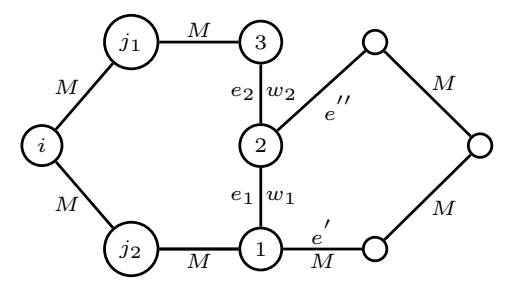

Figure 15: $w_{1}<w_{2} \leq M$ and $e^{\prime}$ (resp. $\left.e^{\prime \prime}\right)$ may coincide with $e_{m}$ (resp. $e_{2}$ ). $e^{\prime} \in C_{1}$ (resp. $\left.e^{\prime \prime} \in C_{1}\right)$ then $w\left(e^{\prime}\right)=M$ (resp. $\left.w\left(e^{\prime \prime}\right)=w_{2}\right)$. Otherwise, as $w_{1}<w_{2} \leq M$, the Star condition applied to $\left\{e_{1}, e_{m}, e^{\prime}\right\}$ (resp. $\left.\left\{e_{1}, e_{2}, e^{\prime \prime}\right\}\right)$ implies $w\left(e^{\prime}\right)=M$ (resp. $\left.w\left(e^{\prime \prime}\right)=w_{2}\right)$. Then the Cycle condition applied to $C_{2}$ and Lemma 13 imply $w_{1}<w\left(e^{\prime \prime}\right) \leq M$ and all other edges in $C_{2}$ have weight $M$. If $w\left(e^{\prime \prime}\right)<M$ and $e^{\prime \prime}=e_{2}$ then $e_{1}$ and $e_{2}$ are two non-maximum common edges, contradicting the Adjacent Cycles condition. Otherwise $e_{1}$ is a unique non-maximum edge common to $C_{1}$ and $C_{2}$, but all edges adjacent 
to $e_{1}$ have a weight strictly greater than $w_{1}$, contradicting the Adjacent Cycles condition.

Let us now assume $\sigma(A)>\sigma(B)$. As $B$ is connected and as $A \subseteq B$ and $\sigma(A)>\sigma(B)$, there is an edge $e$ in $E(B)$ of weight $\sigma(B)$ linked by a path to $C_{1}$ as represented in Figure 16, but it contradicts the Pan condition.

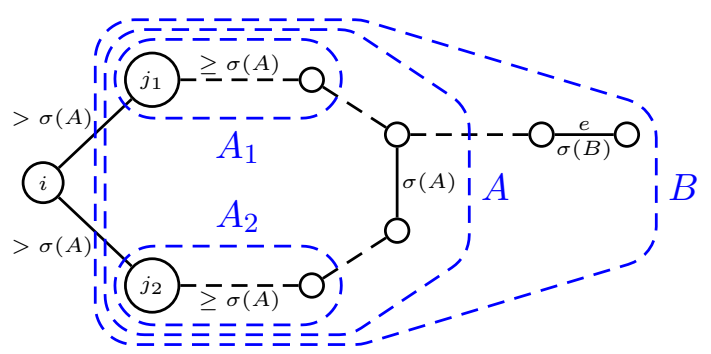

Figure 16: $\left|V\left(C_{1}\right)\right| \geq 3$ and $\sigma(A)>\sigma(B)$.

Hence we can always assume $A_{j} \subseteq B_{j}$, for all $j, 1 \leq j \leq k \leq l$. Moreover, if $\sigma(A)>\sigma(B)$, we can assume $A \subseteq B_{1}$ as $A$ is connected, and therefore we necessarily have $k=1$.

Remark 5. If $\sigma(A)=\sigma(B)$ and if the Path condition is satisfied then the existence of an edge $e \in E(B, i) \backslash E(A, i)$ such that $w(e)<\sigma(A)$ also implies $k=1$. Moreover, by the Star condition all edges $e$ in $E(A, i)$ have same weight $w(e)>\sigma(A)$ and are incident to the same subset $A_{1} \in \mathcal{P}_{\min }(A)$.

Proof of Theorem 15. We have already proved that the conditions are necessary. We now prove they are sufficient. Let $(N, v)$ be a given $\mathcal{F}$-convex game. According to Theorem 2, we have to prove that, for all $i \in N$, for all $A \subseteq B \subseteq N \backslash\{i\}$ and $A, B, A \cup\{i\} \in \mathcal{F}$, we have:

$$
\bar{v}(B \cup\{i\})-\bar{v}(B) \geq \bar{v}(A \cup\{i\})-\bar{v}(A) .
$$

As $A \cup\{i\}$ is connected, there exists an edge $e=\{i, j\}$ with $j \in A$. As $E(A, i) \subseteq E(B, i)$, we have $\sigma(A, i) \geq \sigma(B, i)$. Using Lemma 19, we have several cases to consider.

Case $1 \sigma(A)=\sigma(B)>\sigma(A, i)$.

Case 1.1 Let us first assume there exists an edge $e \in E(A, i)$ such that $w(e)>\sigma(A, i)$. Then $\mathcal{P}_{\min }(A \cup\{i\})=\{A \cup\{i\}\}$ (resp. $\mathcal{P}_{\min }(B \cup\{i\})=$ $\{B \cup\{i\}\})$ as edges in $E(A)$ (resp. $E(B))$ and $e$ are not deleted. Hence (31) is equivalent to $v(B \cup\{i\})-\bar{v}(B) \geq v(A \cup\{i\})-\bar{v}(A)$. This last inequality is satisfied as Lemma 18 implies $v(B)-\bar{v}(B) \geq v(A)-\bar{v}(A)$ and the $\mathcal{F}$ convexity of $v$ implies $v(B \cup\{i\})-v(B) \geq v(A \cup\{i\})-v(A)$.

Case 1.2 Let us now assume that for all $e \in E(A, i), w(e)=\sigma(A, i)$. We consider several subcases.

Case 1.2.1 Let us assume that there exists an edge $e \in E(B, i)$ such that $w(e)>\sigma(B, i)$. Then $\mathcal{P}_{\min }(A \cup\{i\})=\{A,\{i\}\}$ (all edges in $E(A, i)$ are 
deleted) and $\mathcal{P}_{\min }(B \cup\{i\})=\{B \cup\{i\}\}$ (edges in $E(B)$ and $e$ are not deleted). Then (31) is equivalent to $v(B \cup\{i\})-\bar{v}(B) \geq v(A)+v(i)-\bar{v}(A)$. This last inequality is satisfied as Lemma 18 implies $v(B)-\bar{v}(B) \geq v(A)-\bar{v}(A)$ and the superadditivity of $v$ implies $v(B \cup\{i\})-v(B) \geq v(i)$.

Case 1.2.2 Let us now assume that for all $e \in E(B, i), w(e)=\sigma(B, i)$. Then $\mathcal{P}_{\min }(A \cup\{i\})=\{A,\{i\}\}$ and $\mathcal{P}_{\min }(B \cup\{i\})=\{B,\{i\}\}$. Therefore (31) is equivalent to $v(B)-\bar{v}(B) \geq v(A)-\bar{v}(A)$. Lemma 18 implies that this inequality is satisfied.

Case $2 \sigma(A, i) \geq \sigma(A) \geq \sigma(B)$.

If $w(e)=\sigma(A)$ for all $e \in E(A, i)$ then $\mathcal{P}_{\min }(A \cup\{i\})=\left\{\mathcal{P}_{\min }(A),\{i\}\right\}$, and (31) is equivalent to $\bar{v}(B \cup\{i\})-\bar{v}(B) \geq \bar{v}(A)+v(i)-\bar{v}(A)=v(i)$. Proposition 4 implies the superadditivity of $\bar{v}$ and therefore the last inequality is satisfied. Otherwise, let $A_{1}, A_{2}, \ldots, A_{k}$ (resp. $B_{1}, B_{2}, \ldots, B_{l}$ ) be the components of $\mathcal{P}_{\min }(A)$ (resp. $\left.\mathcal{P}_{\min }(B)\right)$ connected to $i$ by edges in $E(A, i)$ (resp. $E(B, i)$ ) with weights strictly greater than $\sigma(A)$ (following Lemma 20, we have $k=1$, if $\sigma(A)>\sigma(B)$ ). Then, setting $\tilde{A}=A_{1} \cup A_{2} \cup \ldots \cup A_{k}$ and $\tilde{B}=B_{1} \cup B_{2} \cup \ldots \cup B_{k}$, (31) is equivalent to:

$$
v\left(\tilde{B} \cup B_{k+1} \cup \cdots \cup B_{l} \cup\{i\}\right)-\sum_{j=1}^{l} v\left(B_{j}\right) \geq v(\tilde{A} \cup\{i\})-\sum_{j=1}^{k} v\left(A_{j}\right) .
$$

Applying Lemma 20, we can assume $A_{j} \subseteq B_{j}$, for all $j, 1 \leq j \leq k \leq l$. Then, applying Corollary 17 to $\tilde{A} \cup\{i\}$ and $\tilde{B} \cup\{i\}$ with $\mathcal{P}(\tilde{A} \cup\{i\})=$ $\left\{A_{1}, \ldots, A_{k},\{i\}\right\}$ and $\mathcal{P}(\tilde{B} \cup\{i\})=\left\{B_{1}, \ldots, B_{k},\{i\}\right\}$, we get $v(\tilde{B} \cup\{i\})-$ $\sum_{j=1}^{k} v\left(B_{j}\right) \geq v(\tilde{A} \cup\{i\})-\sum_{j=1}^{k} v\left(A_{j}\right)$. The superadditivity of $v$ implies $v\left(\tilde{B} \cup B_{k+1} \cup \ldots \cup B_{l} \cup\{i\}\right)-\sum_{j=1}^{l} v\left(B_{j}\right) \geq v(\tilde{B} \cup\{i\})-\sum_{j=1}^{k} v\left(B_{j}\right)$. These last inequalities imply (32).

Remark 6. As the example given in Figure 2 of Section 3 satisfies the Path condition, it satisfies inheritance of $\mathcal{F}$-convexity by Theorem 15 .

We finally get general necessary and sufficient conditions for inheritance of $\mathcal{F}$-convexity with $\mathcal{P}_{\text {min }}$.

Corollary 21. Let $G=(N, E, w)$ be a weighted graph. The following properties are equivalent:

1) For every unanimity game $\left(N, u_{S}\right)$, the $\mathcal{P}_{\min }$-restricted game $\left(N, \overline{u_{S}}\right)$ is $\mathcal{F}$-convex.

2) For every superadditive and $\mathcal{F}$-convex game $(N, v)$, the $\mathcal{P}_{\text {min }}$-restricted game $(N, \bar{v})$ is $\mathcal{F}$-convex.

3) For all $A, B \in \mathcal{F}$ such that $A \cap B \in \mathcal{F}, \mathcal{P}_{\min }(A \cap B)=\left\{A_{j} \cap B_{k} \mid A_{j} \in\right.$ $\mathcal{P}_{\min }(A), B_{k} \in \mathcal{P}_{\min }(B)$ s.t. $\left.A_{j} \cap B_{k} \neq \emptyset\right\}$. 
4) For all $i \in N$, for all $A \subset B \subseteq N \backslash\{i\}$ with $A, B, A \cup\{i\} \in \mathcal{F}$, and for all $A^{\prime} \in \mathcal{P}_{\min }(A \cup\{i\}), \mathcal{P}_{\text {min }}(B)_{\mid A^{\prime}}=\mathcal{P}_{\text {min }}(A)_{\mid A^{\prime}}$.

Proof. Let us assume 1) is satisfied. Then Propositions 7, 10, 11, 12 and 14 imply that the conditions of Theorem 15 are satisfied and therefore 2) is satisfied. Obviously 2) implies 1). As we consider the correspondence $\mathcal{P}_{\min }$, Proposition 4 implies that we have inheritance of superadditivity. Then by Theorem 3,1 ) is equivalent to 3 ) and 4 ).

\subsection{Equivalence between cycle-complete condition for $G$ and Adjacent Cycle condition for $G^{\prime}$.}

We have established in Section 4 that the Myerson restricted game on a graph $G$ corresponds to a restriction of the $\mathcal{P}_{\text {min-restricted game for a par- }}$ ticular weighted graph $G^{\prime}$. We now prove that the Path, Star, Cycle, and Pan conditions are always satisfied on $G^{\prime}$, and that the Adjacent Cycles condition is satisfied on $G^{\prime}$ if and only if $G$ is cycle-complete.

Proposition 22. Let $G=(N, E)$ be a graph and $G^{\prime}=\left(N^{\prime}, E^{\prime}, w\right)$ be the weighted connected graph obtained from $G$ by the procedure of Section 4. $G^{\prime}$ satisfies the Path, Star, Cycle and Pan conditions.

Proof. Let $\gamma=\left\{1, e_{1}, 2, e_{2}, \ldots, m, e_{m}, m+1\right\}$ be an elementary path in $G^{\prime}$. If $w_{1}=w_{m}=\frac{1}{2}$, then $e_{1}$ and $e_{m}$ are incident to $s$. We necessarily have $2=s$ and $m=2$ otherwise $\gamma$ would form a cycle. Then $\gamma$ trivially satisfies Path condition. Otherwise, we can assume w.l.o.g. $w_{1}=1$ and as $w_{i}=1$ or $\frac{1}{2}$ for all $i, 1 \leq i \leq m$, we trivially have $w_{i} \leq \max \left(w_{1}, w_{m}\right)=1$ for all $i, 1 \leq i \leq m$. Hence Path condition is satisfied. Let us now consider a star $\left\{e_{1}, e_{2}, e_{3}\right\}$ with $e_{1}=\{1,2\}, e_{2}=\{1,3\}$, and $e_{3}=\{1,4\}$. We can assume $w_{1} \leq w_{2} \leq w_{3}$. As $w(e)=\frac{1}{2}$ if and only if $e$ is incident to $s$ we only have three possible cases represented in Figures 17. Therefore Star condition is obviously satisfied. Let us now consider a simple cycle $C_{m}=\left\{1, e_{1}, 2, e_{2}, \ldots, m, e_{m}, 1\right\}$. If $s \notin$

(a)

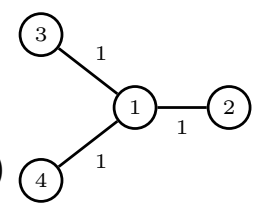

(b)

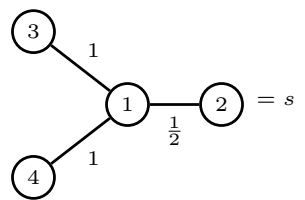

(c)

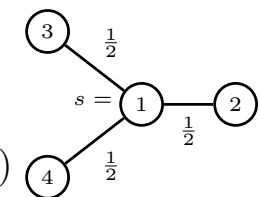

Figure 17: (a) : $w_{1}=w_{2}=w_{3}=1$, (b) : $w_{1}=\frac{1}{2}<1=w_{2}=w_{3}$, (c) : $w_{1}=w_{2}=w_{3}=\frac{1}{2}$.

$V\left(C_{m}\right)$ then $w_{1}=w_{2}=\cdots=w_{m}=1$. Otherwise, we can assume w.l.o.g. $s=2$ and then $w_{1}=w_{2}=\frac{1}{2}<1=w_{3}=w_{4}=\cdots=w_{m}$ as represented in Figure 18. Hence the Cycle condition is also satisfied. Let us consider a simple cycle $C_{m}=\left\{e_{1}, e_{2}, \ldots, e_{m}\right\}$ and an elementary path $P_{r}$ such that 


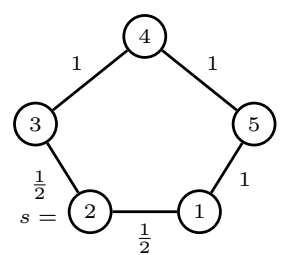

Figure 18: $w_{1}=w_{2}=\frac{1}{2}<1=w_{3}=\cdots=w_{m}$.

$\left|V\left(C_{m}\right) \cap V\left(P_{r}\right)\right|=1$. If $s \notin V\left(C_{m}\right)$ then $w_{1}=w_{2}=\cdots=w_{m}=1$. Otherwise $\min _{1 \leq k \leq m} w_{k}=\frac{1}{2}$. We have $w(e) \in\left\{\frac{1}{2}, 1\right\}$ for all $e \in E$. Therefore there is no edge $e$ in $P_{r}$ with $w(e)<\min _{1 \leq k \leq m} w_{k}$. Hence Pan condition is satisfied (in fact this condition is not relevant).

Proposition 23. Let $G=(N, E)$ be a graph and $G^{\prime}=\left(N^{\prime}, E^{\prime}, w\right)$ be the weighted connected graph obtained from $G$ by the procedure of Section 4. $G$ is cycle-complete if and only if $G^{\prime}$ satisfies the Adjacent Cycles condition.

Proof. By contradiction, let us assume there is a cycle $C_{m}=\left\{1, e_{1}, 2, e_{2}, \ldots\right.$, $\left.e_{m}, 1\right\}$ in $G$ which is not complete. After renumbering if necessary, we can assume that $\{1, j\} \notin E$ with $j \in\{3, \ldots, m-1\}$ and $j$ as small as possible. If $j \geq 4$, then $\tilde{e}=\{1, j-1\}$ is a chord of $C_{m}$. We can replace $C$ by the smaller cycle $\tilde{C}=\left\{1, \tilde{e}, j-1, e_{j-1}, j, e_{j}, \ldots, m, e_{m}, 1\right\}$ (which is not complete) as represented in Figure 19. Hence we can assume $j=3$. We can assume

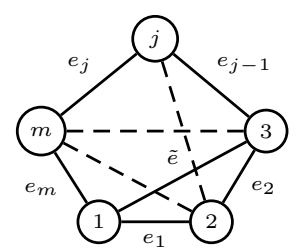

Figure 19: $\{1, j\} \notin E(C)$ with $j \geq 4$.

that there is no chord $\{k, l\}$, with $k \neq 2$ and $l \neq 2$, otherwise we can replace $C$ by a smaller cycle. Hence any chord of $C$ is incident to 2 . Let us now consider the two adjacent cycles $\tilde{C}$ and $\tilde{C}^{\prime}$ in $G^{\prime}$, obtained adding the edges $\tilde{e}_{1}=\{s, 1\}$ and $\tilde{e}_{3}=\{s, 3\}, \tilde{C}=\left\{s, \tilde{e}_{1}, 1, e_{1}, 2, e_{2}, 3, \tilde{e}_{3}, s\right\}$, and $\tilde{C}^{\prime}=\left\{s, \tilde{e}_{3}, 3, e_{3}, 4, \ldots, m, e_{m}, 1, \tilde{e}_{1}, s\right\}$, as represented in Figure 20. Then $\tilde{C}$ and $\tilde{C}^{\prime}$ have no maximum weight chord. $\tilde{C}$ has only one chord $\{s, 2\}$ of weight $\frac{1}{2}$. We have $|\tilde{C}|=4$ and $\left|\tilde{C}^{\prime}\right| \geq 4$, and $\tilde{e}_{1}, \tilde{e}_{3}$ are two common non-maximum weight edges of $\tilde{C}$ and $\tilde{C}^{\prime}$. Hence $\tilde{C}$ and $\tilde{C}^{\prime}$ contradict the Adjacent Cycles condition in $G^{\prime}$.

Conversely assume that the Adjacent Cycles condition is not satisfied in $G^{\prime}$. Let $\tilde{C}$ and $\tilde{C}^{\prime}$ be two adjacent cycles in $G^{\prime}$ satisfying the conditions of the Adjacent Cycles condition except that they have two common nonmaximum weight edges $\tilde{e}_{1}$ and $\tilde{e}_{3}$ (of weight $\frac{1}{2}$ ) and $|V(\tilde{C})|,\left|V\left(\tilde{C}^{\prime}\right)\right| \geq 4$. 


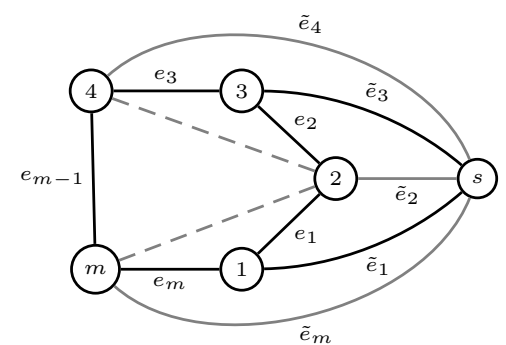

Figure 20: $\tilde{C}$ and $\tilde{C}^{\prime}$ in $G^{\prime}$.

Then $\tilde{e}_{1}$ and $\tilde{e}_{3}$ are necessarily incident to $s$ which is a common vertex of $\tilde{C}$ and $\tilde{C}^{\prime}$. By assumption $\tilde{C}$ and $\tilde{C}^{\prime}$ have no maximum weight chord (of weight 1 ), and no common chord. Moreover $\tilde{C}$ can have at most one nonmaximum weight chord. As $s$ is linked to all the other vertices we necessarily have $|V(\tilde{C})|=4$ (otherwise $\tilde{C}$ has more than one chord). We set $\tilde{C}=$ $\left\{s, \tilde{e}_{1}, 1, e_{1}, 2, e_{2}, 3, \tilde{e}_{3}, s\right\}$, and $\tilde{C}^{\prime}=\left\{s, \tilde{e}_{3}, 3, e_{3}, 4, \ldots, m, e_{m}, 1, \tilde{e}_{1}, s\right\}$, as represented in Figure 20. Then we consider the cycle $C=\left\{1, e_{1}, 2, e_{2}, 3, e_{3}, \ldots\right.$, $\left.m, e_{m}, 1\right\} \quad\left(=\left(\tilde{C}^{\prime} \backslash\{s\}\right) \cup\{2\}\right)$. We have $|V(C)| \geq 4$. Then a chord of $C$ can only be incident to 2 (otherwise it is a chord of $\tilde{C}^{\prime}$ of maximum weight, contradicting the assumption). Therefore $C$ is a non-complete cycle and $G$ is not cycle-complete. Let us now observe that the second part of the Adjacent Cycles condition is always satisfied. Let $\tilde{C}$ and $\tilde{C}^{\prime}$ be two adjacent cycles in $G^{\prime}$ satisfying the conditions of the Adjacent Cycles condition and with one common non-maximum weight edge $e_{1}$. Then $e_{1}$ is incident to $s$ and therefore $s$ is a common vertex of $\tilde{C}$ and $\tilde{C}^{\prime}$. As all edges incident to $s$ have weight $\frac{1}{2}$, we always have $w_{1}=w_{2}=w_{2}^{\prime}=\frac{1}{2}$ (with the notations of the Adjacent Cycles condition).

Finally, we get the following result which also gives the graph characterization established in (van den Nouweland and Borm, 1991) for inheritance of convexity with Myerson restricted game.

Corollary 24. Let $G=(N, E)$ be a graph and $G^{\prime}=\left(N^{\prime}, E^{\prime}, w\right)$ be the weighted connected graph obtained from $G$ by the procedure of Section 4 . Let us consider a zero-normalized game $\left(N^{\prime}, v\right)$. Let $\mathcal{F}$ be the family of connected subsets of $N^{\prime}$. Then, the following conditions are equivalent:

1. There is inheritance of $\mathcal{F}$-convexity from $\left(N^{\prime}, v\right)$ to the $\mathcal{P}_{\min }$-restricted game $\left(N^{\prime}, \bar{v}\right)$.

2. $G^{\prime}$ satisfies the Adjacent Cycles condition.

3. There is inheritance of convexity from $(N, v)$ to $\left(N, v^{M}\right)$.

4. $G$ is cycle-complete. 
Proof. 1 and 2 are equivalent by Theorem 15 and Proposition 22. 1 and 3 are equivalent by Corollary 6.2 and 4 are equivalent by Proposition 23 .

It is interesting to observe that to establish the inheritance of convexity from $\left(N^{\prime}, v\right)$ to $\left(N^{\prime}, \bar{v}\right)$ with $G^{\prime}$ we only need to verify the Adjacent Cycles condition. Moreover, to verify the Adjacent Cycles condition it is sufficient (as observed in the proof of Proposition 23) to verify the non-existence of a pair of adjacent cycles $C$ and $C^{\prime}$ with $|V(C)|=4$ and $\left|V\left(C^{\prime}\right)\right| \geq 4$ and having two common non-maximum weight edges (and satisfying the conditions 1 , 2, 3, 4 of the Adjacent Cycles condition).

\subsection{A description of graphs satisfying the necessary condi- tions for inheritance of $\mathcal{F}$-convexity}

We finally describe more precisely the connected graphs satisfying the necessary conditions defined in Section 5.1.

Proposition 25. Let $G=(N, E, w)$ be a weighted connected graph. Let $E_{1}$ be the set of minimum weight edges in $E$ and $N_{1}$ be the set of their endvertices in $N$. If the edge-weight function $w$ satisfies the Star, Path, Cycle, Pan, and Adjacent Cycles conditions then:

1) If an elementary path $\gamma$ in $G$ has its first edge in $E_{1}$ then the edge-weights of $\gamma$ are non decreasing.

2) $G_{1}=\left(N_{1}, E_{1}\right)$ is a connected subgraph.

3) If $\left|E_{1}\right|=1$ then there exists at most one chordless cycle $\tilde{C}$ with $E(\tilde{C}) \cap$ $E_{1} \neq \emptyset$. For all cycle $C_{m}=\left\{1, e_{1}, 2, e_{2}, \ldots, m, e_{m}, 1\right\}$ with $m \geq 3$ and $E\left(C_{m}\right) \cap E_{1}=\emptyset$, either $C_{m}$ has constant edge-weights or $\sigma(N)<w_{1}=$ $w_{2}<w_{3}=\cdots=w_{m}=M$, where $M=\max _{e \in E\left(C_{m}\right)} w(e)$. In this last case $\{1,3\}$ is a maximum weight chord of $C_{m}$ and if $2 \notin N_{1}$ then 2 is a cut vertex ${ }^{6}$ of $G$.

4) If $\left|E_{1}\right| \geq 2$ then for all cycle $C_{m}=\left\{1, e_{1}, 2, e_{2}, \ldots, m, e_{m}, 1\right\}$ with $m \geq 3$ we have $\sigma(N) \leq w_{1}=w_{2} \leq w_{3}=\cdots=w_{m}=M$. If $\sigma(N)<w_{1}=$ $w_{2}<M$, then $\{1,3\}$ is a maximum weight chord and if $2 \notin N_{1}$ then 2 is a cut vertex of $G$. If $\sigma(N)=w_{1}=w_{2}<M$ and if there exists an edge in $E_{1}$ non incident to 2 , then 2 is a cut vertex of $G$ (but $\{1,3\}$ is not necessarily a chord).

We give an example of a graph satisfying Proposition 25 with $\left|E_{1}\right|=1$ (resp. $\left|E_{1}\right| \geq 2$ ) in Figure 21 (resp. Figure 22).

Proof. 1) Immediately results from Path condition.

\footnotetext{
${ }^{6} \mathrm{~A}$ cut vertex (or articulation point) in a graph is a vertex the removal of which disconnects the graph.
} 


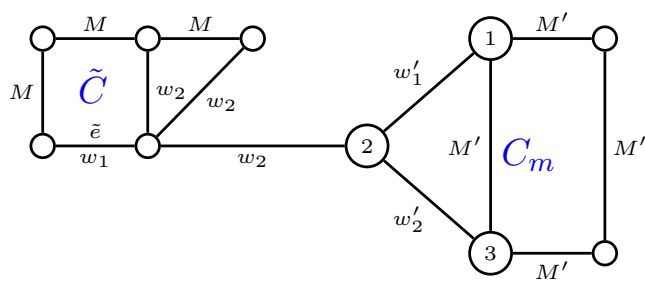

Figure 21: $E_{1}=\{\tilde{e}\}, w(\tilde{e})=w_{1}<w_{2} \leq M$ in $\tilde{C}$ and $w_{1}^{\prime}=w_{2}^{\prime}<M^{\prime}$ in $C_{m}$.

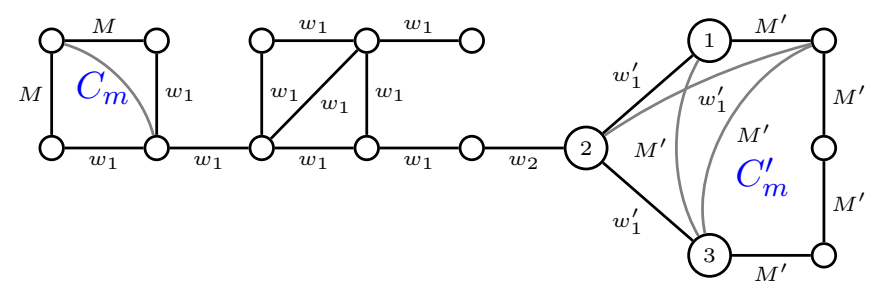

Figure 22: $\left|E_{1}\right| \geq 2, \sigma(N)=w_{1}<M$, and $w_{1} \leq w_{2}<w_{1}^{\prime}=w_{2}^{\prime}<M^{\prime}$.

2) Let us consider $v^{\prime}$ and $v^{\prime \prime}$ in $N_{1}$. By definition $v^{\prime}$ and $v^{\prime \prime}$ are endvertices of edges $e^{\prime}$ and $e^{\prime \prime}$ in $E_{1}$ such that $w\left(e^{\prime}\right)=w\left(e^{\prime \prime}\right)=\sigma(N)$. If $e^{\prime}=e^{\prime \prime}$ or if $e^{\prime}$ and $e^{\prime \prime}$ are adjacent, then $e^{\prime} \cup e^{\prime \prime}$ corresponds to a path in $G_{1}$ linking $v^{\prime}$ to $v^{\prime \prime}$. Otherwise let $\gamma$ be a shortest path in $G$ linking $e^{\prime}$ to $e^{\prime \prime}$. Then, Path condition applied to $\gamma^{\prime}=e^{\prime} \cup \gamma \cup e^{\prime \prime}$ implies $w(e) \leq \max \left(w\left(e^{\prime}\right), w\left(e^{\prime \prime}\right)\right)=\sigma(N)$ and therefore $w(e)=\sigma(N)$ for all edge $e \in \gamma$. Hence $\gamma^{\prime}$ is a path from $v^{\prime}$ to $v^{\prime \prime}$ in $G_{1}$.

3) Let $\tilde{e}$ be the unique edge in $E_{1}$. By contradiction, let us assume that $\tilde{e}$ is a common edge of two cycles $C$ and $C^{\prime}$ without chords. Then, the Adjacent Cycles condition implies that some edge of $C$ or $C^{\prime}$ adjacent to $\tilde{e}$ has a weight equal to $w(\tilde{e})$ and therefore $\left|E_{1}\right| \geq 2$, a contradiction. For a cycle $C_{m}$ such that $\tilde{e} \notin E\left(C_{m}\right)$, the Pan condition implies the result. In particular, if $2 \notin N_{1}$ then $e_{1}$ is not incident to $C_{m}$ and every path $\gamma$ linking $\tilde{e}$ to $C_{m}$ has to end at vertex 2 . Therefore if we delete vertex 2 the graph is disconnected.

4) Cycle condition applied to $C_{m}=\left\{1, e_{1}, 2, e_{2}, \ldots, m, e_{m}, 1\right\}$ implies $w_{1} \leq w_{2} \leq w_{3}=\cdots=w_{m}=M$, after renumbering if necessary, and that all chords of $C_{m}$ have weight $w_{2}$ or $M$. If $\left\{e_{1}, e_{2}\right\} \nsubseteq E_{1}$ then there necessarily exists an edge $e \in E_{1} \backslash E\left(C_{m}\right)$ and Pan condition implies either $\sigma(N)<w_{1}=w_{2}=M$ or $\sigma(N)<w_{1}=w_{2}<M$. In this last case $\{1,3\}$ is a maximum weight chord of $C_{m}$ and if $2 \notin N_{1}$ then 2 is an articulation point. If $\left\{e_{1}, e_{2}\right\} \subseteq E_{1}$ then $\sigma(N)=w_{1}=w_{2} \leq M$. Let us assume $\sigma(N)=w_{1}=w_{2}<M$ and that there exists $e \in E_{1}$ non incident to 2. No path $\gamma$ can link $e$ to some vertex $j \in V\left(C_{m}\right) \backslash\{2\}$, otherwise it contradicts Path condition applied to the path $\gamma^{\prime}$ linking 
$e$ to $e_{1}$ or $e_{2}$ passing through vertex $j$ as represented in Figure 23 (note that if $j \neq 1$ and $j \neq 3$ then $\gamma^{\prime}$ contains an edge in $C_{m}$ of weight $M$, otherwise Star condition implies that the edge of $\gamma$ incident to 1 or 3 has weight $M$ ). 2 is not necessarily a cut vertex if all edges of $E_{1}$ are incident to it, as it is shown in Figure 24.

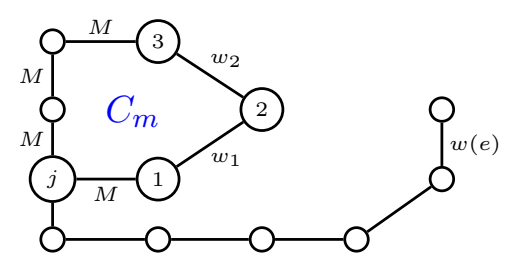

Figure 23: $\left|E_{1}\right| \geq 2, \sigma(N)=w_{1}=w_{2}=w(e)<M$.

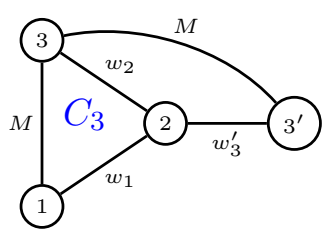

Figure 24: $E_{1}=\left\{e_{1}, e_{2}, e_{3}^{\prime}\right\}, \sigma(N)=w_{1}=w_{2}=w_{3}^{\prime}<M$.

\section{Inheritance of convexity for $\mathcal{P}_{\min }$}

We finally present some results on inheritance of classical convexity for $\mathcal{P}_{\text {min }}$ established in a forthcoming paper Skoda (2017). The first result highlights a major difference between graphs satisfying inheritance of $\mathcal{F}$-convexity and the ones satisfying inheritance of convexity. Whereas inheritance of $\mathcal{F}$-convexity allows an arbitrary number of edge-weights, inheritance of convexity restricts the number of edge-weights to at most three.

Proposition 26. Let $G=(N, E, w)$ be a weighted graph. Let us assume that for every unanimity game $\left(N, u_{S}\right)$, the $\mathcal{P}_{\min }$-restricted game $\left(N, \overline{u_{S}}\right)$ is convex. Then the edge-weights have at most three different values $\sigma_{1}<\sigma_{2}<$ $\sigma_{3}$. Moreover, if $\left|E_{1}\right| \geq 2$ (where $E_{1}=\left\{e \in E ; w(e)=\sigma_{1}\right\}$ ), then the edge-weights have at most two different values $\sigma_{1}<\sigma_{2}$.

Proposition 26 implies that if the edge-weights have three different values, then there is only one edge of minimum weight $\sigma_{1}$. In contrast, the number of different edge-weights has no incidence on the number of minimum weight edges in the case of inheritance of $\mathcal{F}$-convexity 
The following theorems give characterizations of graphs satisfying inheritance of convexity. Of course, the five conditions for inheritance of $\mathcal{F}$ convexity have to be satisfied to have inheritance of convexity, but these characterizations do not refer to the conditions established in Section 5. Actually, these characterizations rely on stronger and more direct conditions on the graph, in particular on the incidence of edges according to their weights. These conditions imply that the Path, Star, Cycle, Pan, and Adjacent Cycles conditions are automatically satisfied. Nevertheless, the conditions for inheritance of $\mathcal{F}$-convexity are required to prove Proposition 26 and to establish that the graphs described by the following characterizations are the only ones satisfying inheritance of convexity (cf. Skoda (2017)).

Let us first consider graphs with two edge-weights.

Theorem 27. Let $G=(N, E, w)$ be a weighted graph. Let us assume that the edge-weights have only two different values $\sigma_{1}<\sigma_{2}$. Let us consider $E_{1}=\left\{e \in E ; w(e)=\sigma_{1}\right\}$ and $E_{2}=\left\{e \in E ; w(e)=\sigma_{2}\right\}$. Let us assume $\left|E_{1}\right| \geq 2$. Then there is inheritance of convexity for $\mathcal{P}_{\min }$ if and only if:

1. All edges in $E_{1}$ are incident to the same vertex 1 and all edges in $E_{2}$ are linked to 1 by an edge in $E_{1}$.

2. One of the two following equivalent conditions is satisfied:

(a) There is inheritance of convexity for $\mathcal{P}_{M}$ (Myerson's correspondence) on the subgraph $G_{2}=\left(N, E_{2}\right)$.

(b) $G_{2}=\left(N, E_{2}\right)$ is cycle-complete.

Note that Condition 1 implies that an edge in $E_{2}$ is not incident to 1. Moreover, it implies that the Star, Path, Cycle and Pan conditions are satisfied. Adding Condition 2, the Adjacent Cycles condition is also satisfied. We give in Figure 25 an example of a graph satisfying the conditions of Theorem 27. We can also observe that the weighted graphs obtained by

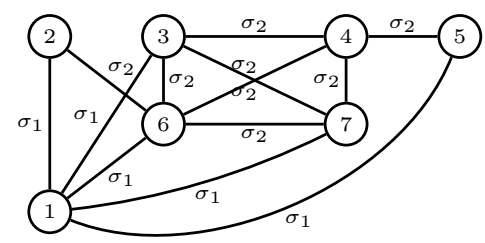

Figure 25: Every edge of weight $\sigma_{2}$ is linked to 1 by an edge of weight $\sigma_{1}$ and the cycle induced by $\{3,4,6,7\}$ is complete.

the procedure of Section 4 satisfy Condition 1. Then Theorem 27 implies Corollary 6 .

Theorem 28. Let $G=(N, E, w)$ be a weighted graph. Let us assume that the edge-weights have only two different values $\sigma_{1}<\sigma_{2}$. Let us assume 
$\left|E_{1}\right|=1$. Let $e_{1}$ be the unique edge in $E_{1}$. Then there is inheritance of convexity for $\mathcal{P}_{\min }$ if and only if:

1. There exists at most one chordless cycle containing $e_{1}$.

2. For every cycle $C$ with constant weight $\sigma_{2}$ either $C$ is complete or all vertices of $C$ are linked to the same end vertex of $e_{1}$.

As $\left|E_{1}\right|=1$, the Star, Path, Cycles, and Pan conditions are satisfied. By Condition 1 the Adjacent Cycles condition is also satisfied. We give in Figure 26 an example of a graph satisfying the conditions of Theorem 28.

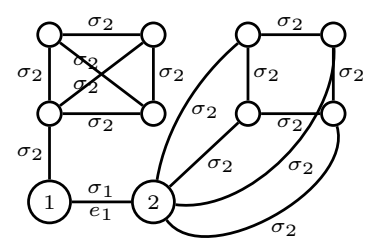

Figure 26: Either a cycle with constant weight is complete or all its vertices are linked to one end-vertex of $e_{1}$.

We now consider graphs with three different edge-weights.

Theorem 29. Let $G=(N, E, w)$ be a weighted graph. Let us assume that the edge-weights have three different values $\sigma_{1}<\sigma_{2}<\sigma_{3}$. Then there is inheritance of convexity for $\mathcal{P}_{\min }$ if and only if:

1. There exists only one edge $e_{1}=\{1,2\}$ of weight $\sigma_{1}$.

2. An edge in $E \backslash\left\{e_{1}\right\}$ has weight $\sigma_{2}$ if and only if it is incident to the same end-vertex 2 of $e_{1}$.

3. Every edge of weight $\sigma_{3}$ is connected to 2 by $e_{1}$ or by an edge of weight $\sigma_{2}$.

4. There exists at most one (chordless) cycle $\tilde{C}_{m}$ with $m=3$ or 4 containing $e_{1}$.

5. Every cycle $C_{m}$ which does not contain $e_{1}$ is complete.

6. If a cycle $C_{m}$ does not contain $e_{1}$ and if $1 \in V\left(C_{m}\right)$, then $m=3$ and such a triangle is unique (if it exists) and is adjacent to a unique triangle $\tilde{C}_{3}$ which contains the edge $e_{1}$.

Note that Conditions 1, 2, and 3 straightforwardly imply the Star, Path, and Cycle conditions. Adding Condition 4 (resp. Condition 5) they also imply the Adjacent Cycles (resp. Pan) condition. We give in Figure 27 an example of a graph satisfying the conditions of Theorem 29 . 


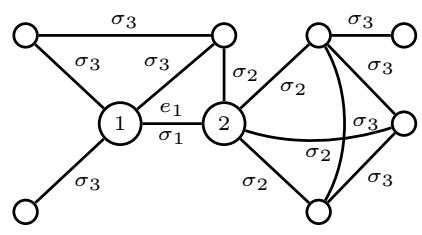

Figure 27: $\sigma_{1}<\sigma_{2}<\sigma_{3}$.

Some parts of the proofs of the characterizations given in Theorems 27 and 28 for graphs with two edge-weights are easily obtained as they can be reduced to cycle-completeness analysis. Contrary to what one might expect, there is no such reduction for the case of graphs with exactly three different edge-weights. Moreover, despite the restriction to only three edge-weights, the proof of the characterization given in Theorem 29 remains difficult in comparison with the ones for inheritance of $\mathcal{F}$-convexity where an arbitrary number of edge-weights is considered.

Besides the number of possible edge-weights, we finally state some other major differences between graphs satisfying inheritance of convexity and graphs satisfying inheritance of $\mathcal{F}$-convexity. These differences can be observed by comparing the previous characterizations with the description of graphs given in Section 5.4. The distance (i.e., the minimum number of edges) between two vertices in a graph satisfying inheritance of convexity is less or equal to 4 , except when there are two edge-weights $\sigma_{1}<\sigma_{2}$ with $\left|E_{1}\right|=1$. In this last case the distance is not limited (except by $n-1$ ) as for graphs satisfying inheritance of $\mathcal{F}$-convexity. Cycle-completeness is never necessary for inheritance of $\mathcal{F}$-convexity whereas cycle-completeness of certain cycles is required for inheritance of convexity.

\section{Conclusion}

Our main result gives necessary and sufficient conditions for inheritance of $\mathcal{F}$-convexity with the correspondence $\mathcal{P}_{\min }$. This work also constitutes a first step for other correspondences. In particular, the correspondence $\mathcal{P}_{G}$ associated with the strength of a graph presented in (Grabisch and Skoda, 2012), which gives natural partitions, coincides with $\mathcal{P}_{\min }$ on cycle-free graphs. Hence the Star and Path conditions restricted to induced stars and paths are also valid for this correspondence and a natural extension of this work could be to find parallel results for $\mathcal{P}_{G}$. Moreover, the inheritance of superadditivity for $\mathcal{P}_{G}$ is not always satisfied and its characterization is not obvious. We could also consider another restricted game $(N, \tilde{v})$ defined by $\tilde{v}(A)=\sum_{l=1}^{p} \bar{v}\left(A_{l}\right)$ for all $A \subseteq N$, where $A_{1}, A_{2}, \ldots, A_{p}$ are the connected components of $A$. Our characterization of inheritance of $\mathcal{F}$-convexity is also valid for $(N, \tilde{v})$. Then, we can address the problem of the extension of Theorem 15 to inheritance of convexity, but additional conditions are required. 
Borm et al. (1990) introduced arc games. It is well known that, if the communication graph is cycle-free, then there is inheritance of convexity from the underlying game to the corresponding arc game (van den Nouweland and Borm, 1991). We can also define restricted arc games, by substituting to the partition into connected components the correspondence $\mathcal{P}_{\min }$. The question of inheritance of convexity is more difficult as we do not even necessarily have inheritance of superadditivity. All previous correspondences and restricted games are associated with a given graph but we can also consider correspondences without reference to any graph. In this more general setting, we establish in Skoda (2017) an extension of Theorem 3 which provides a characterization of inheritance of convexity for arbitrary correspondences by general set conditions.

\section{Acknowledgments}

The author is grateful to Michel Grabisch for valuable comments and suggestions. The support of the National Research Agency, through MINT (Models of Influence and Network Theory) project, Reference: ANR-09BLAN-0321-01, is gratefully acknowledged.

\section{References}

Algaba, E. (1998). Extensión de juegos definidos en sistemas de conjuntos. $\mathrm{PhD}$ thesis, Univ. of Seville.

Algaba, E., Bilbao, J., Borm, P., and Lopez, J. (2000). The position value for union stable systems. Mathematical Methods of Operations Research, $52: 221-236$.

Algaba, E., Bilbao, J., and Lopez, J. (2001). A unified approach to restricted games. Theory and Decision, 50(4):333-345.

Bilbao, J. M. (2000). Cooperative games on combinatorial structures. Kluwer Academic Publishers, Boston.

Bilbao, J. M. (2003). Cooperative games under augmenting systems. SIAM Journal on Discrete Mathematics, 17(1):122-133.

Borm, P., Owen, G., and Tijs, S. (1990). Values of points and arcs in communication situations. Technical Report 9004, Dept of Mathematics, University of Nijmegen, The Netherlands.

Edmonds, J. and Giles, R. (1977). A min-max relation for submodular functions on graphs. Annals of Discrete Mathematics, 1:185-204. 
Faigle, U. (1989). Cores of games with restricted cooperation. ZOR - Methods and Models of Operations Research, 33(6):405-422.

Faigle, U., Grabisch, M., and Heyne, M. (2010). Monge extensions of cooperation and communication structures. European Journal of Operational Research, 206(1):104-110.

Fujishige, S. (2005). Submodular Functions and Optimization, volume 58 of Annals of Discrete Mathematics. Elsevier, second edition.

Grabisch, M. (2013). The core of games on ordered structures and graphs. Annals of Operations Research, 204(1):33-64.

Grabisch, M. and Skoda, A. (2012). Games induced by the partitioning of a graph. Annals of Operations Research, 201(1):229-249.

Myerson, R. (1977). Graphs and cooperation in games. Mathematics of Operations Research, 2(3):225-229.

Schrijver, A. (2003). Combinatorial Optimization : Polyhedra and Efficiency. Springer-Verlag.

Skoda, A. (2016). Complexity of inheritance of $\mathcal{F}$-convexity for restricted games induced by minimum partitions. Documents de travail du Centre d'Economie de la Sorbonne 2016.55 - ISSN : 1955-611X.

Skoda, A. (2017). Inheritance of convexity for partition restricted games. Discrete Optimization, 25:6 - 27.

Skoda, A. (2017). Inheritance of Convexity for the $\mathcal{P}_{\min }$-Restricted Game. ArXiv e-prints.

van den Nouweland, A. and Borm, P. (1991). On the convexity of communication games. International Journal of Game Theory, 19(4):421-430. 